\title{
Enhanced endoplasmic reticulum and mitochondrial stress in abdominal
}

\author{
aortic aneurysm
}

Running title: Endoplasmic reticulum and mitochondrial stress in AAA.

Miquel Navas-Madroñal ${ }^{1}$, Cristina Rodriguez ${ }^{2,3}$, Modar Kassan ${ }^{4}$, Joan Fité ${ }^{5}$, José R. Escudero, ${ }^{3,5}$, Laia Cañes ${ }^{3,6}$, José Martínez-González ${ }^{3,6}$, Mercedes Camacho ${ }^{1,3}$, María Galán ${ }^{1,3}$.

1. Institut de Recerca del Hospital de la Santa Creu i Sant Pau, Instituto de Investigación Biomédica Sant Pau (IIB Sant Pau), Barcelona, Spain.

2. Institut de Recerca del Hospital de la Santa Creu i Sant Pau-Programa ICCC, IIB Sant Pau, Barcelona, Spain.

3. CIBER de Enfermedades Cardiovasculares, Madrid, Spain.

4. Cardiovascular Division, Department of Medicine and Abboud Cardiovascular Research Center, University of Iowa, Carver College of Medicine, IA City, USA.

5. Servicio de Angiología, Cirugía Vascular y Endovascular, Hospital de la Santa Creu i Sant Pau. Instituto de Investigación Biomédica Sant Pau (IIB Sant Pau), Barcelona, Spain.

6. Instituto de Investigaciones Biomédicas de Barcelona (IIBB-CSIC), IIB Sant Pau, Barcelona, Spain.

\section{Corresponding author:}

Dr. María Galán

Instituto de Investigación Biomédica Sant Pau (IIB Sant Pau)

Hospital de la Santa Creu i Sant Pau

Avda. Sant Antoni Maria Claret, 167

08025 Barcelona. Spain.

TEL: +34 935565622 / 5711; FAX: +3493 5565559

E-mail:mgalana@santpau.cat 


\section{ABSTRACT}

Abdominal aortic aneurysm (AAA) is a degenerative vascular disease with a complex aetiology that remains to be fully elucidated. Clinical management of AAA is limited to surgical repair, while an effective pharmacotherapy is still awaited. Endoplasmic reticulum (ER) stress and mitochondrial dysfunction have been involved in the pathogenesis of cardiovascular diseases, although their contribution to AAA development is uncertain. Therefore, we aimed to determine their implication in AAA and investigated the profile of oxysterols in plasma, specifically 7-ketocholesterol (7-KC), as an ER stress inducer.

In the present study, we determined aortic ER stress activation in a large cohort of AAA patients compared to healthy donors. Higher gene expression of ATF6, IRE-1, XBP-1, CHOP, CRELD2 and SEL1L and greater protein levels of active ATF6, active XBP1 and of the pro-apoptotic protein CHOP were detected in human aneurysmatic samples. This was accompanied by an exacerbated apoptosis, higher reactive oxygen species production and by a reduction in mitochondrial biogenesis in the vascular wall of AAA. The quantification of oxysterols, performed by liquid chromatography-(APCI)-mass spectrometry, showed that levels of $7-\mathrm{KC}$ were significantly higher while those of $7 \alpha$-hydroxycholesterol, 24-hydroxycholesterol and 27-hydroxycholesterol were lower in AAA patients compared to healthy donors. Interestingly, the levels of 7-KC correlate with the expression of ER stress markers.

Our results evidence an induction of ER stress in the vascular wall of AAA patients associated to an increase in circulating 7-KC levels and a reduction in mitochondrial biogenesis suggesting their implication in the pathophysiology of this disease. 
Keywords: abdominal aortic aneurysm, endoplasmic reticulum stress, mitochondrial biogenesis, oxysterols.

\section{ABBREVIATIONS}

AAA: Abdominal aortic aneurysm

7-KC: 7-ketocholesterol

Ang II: Angiotensin II

ECM: Extracellular matrix

ER: endoplasmic reticulum

GAPDH: Glyceraldehyde-3-phosphate dehydrogenase

HC: Hydroxycholesterol

IL: Interleukin

LC-MS: liquid chromatography-mass spectrometry

MMP: Metalloproteinase

NAC: N-acetylcysteine

ROS: Reactive oxygen species

UPR: Unfolding protein response

VSMC: Vascular smooth muscle cells. 


\section{INTRODUCTION}

An abdominal aortic aneurysm (AAA) is defined as a focal dilation of 50 percent greater than the normal average diameter of an aorta. It is a complex disease involving the transmural infiltration of inflammatory cells, the production of reactive oxygen species (ROS), an upregulation and activation of degradative proteases, stimulation of apoptosis and degradation of elastin (1). Even though the histopathological features of AAA are well documented, cellular and molecular mechanisms underlying AAA pathogenesis are not fully understood. Well-defined risk factors that are associated with the development of AAA include advanced age, male gender, smoking, hypertension, Caucasian race, family history, presence of other large vessel aneurysms, and atherosclerosis (2,3). In 2013, according to the Centers for Disease Control, AAA was the 15th leading cause of mortality in the United States in adults between the age of 60 to 64 years old (4). Numerous pharmacologic interventions have been proposed to limit AAA growth but none have provided convincing results in clinical trials (5-9). Therefore, it is imperative to better understand the mechanisms of this important disease to provide new specific drug targets for the treatment of AAA.

We and others have reported the critical role of endoplasmic reticulum (ER) stress in the pathogenesis of hypertension, atherosclerosis, heart failure and other cardiovascular diseases (10-12). Environmental factors such as ischaemia, hypoxia, reactive oxygen species (ROS), aging, and genetic factors disrupt ER function leading to an accumulation of misfolded and unfolded proteins in the ER lumen 
which promotes ER stress (13). Unfolded protein response (UPR) is the adaptive response to ER stress, which is regulated by 3 ER transmembrane proteins triggering three effector branches: inositol requiring enzyme 1 (IRE1), PKR-like ER kinase (PERK), and activating transcription factor 6 (ATF6). In turn, the activation of these effectors trigger transcriptional programs mediated by spliced X-binding protein 1 (XBP-1), activating transcription factor 4 (ATF4) and cleaved ATF6, respectively. Under prolonged ER stress the UPR can lead to cell pathology and apoptosis orchestrated by the C/EBP-Homologous Protein (CHOP). Dysregulation of the UPR pathway has been involved in several diseases such as diabetes, inflammation, neurodegenerative disorders, or cardiovascular disease (14-16). The UPR is often closely linked to mitochondrial stress, ROS responses and autophagy $(17,18)$. Under pathological conditions the crosstalk between mitochondria and the ER promotes mitochondrial ROS generation and mitochondrial dysfunction which is characterized by a decrease of mitochondrial biogenesis and by an increase in oxidative damage $(19,20)$. Therefore, pathways that ameliorate ER stress and / or regulate mitochondrial biogenesis are potential therapeutic targets for diminishing vascular dysfunction and cardiovascular disease. Oxysterols are oxygenated derivatives of cholesterol that are present at low levels in the circulation and have shown to accumulate in the plasma and tissues in some pathologies (21). They have been demonstrated to induce numerous deleterious effects by perturbing cholesterol homeostasis in vascular and immune cells $(21,22)$. Specifically, 7-Ketocholesterol (7-KC) is a well-studied oxysterol formed by the oxidation of cholesterol and cholesterol esters present in lipoprotein deposits. Free 7-KC levels are abundant in the circulation of patients with hypercholesterolaemia and 7-KC also accumulates in atherosclerotic lesions to a greater extent than any 
other oxysterol (23). Moreover, 7-KC is an inducer of ER stress and autophagy in vascular cells $(15,24,25)$.

Due to increasing evidence involving oxysterols in the pathophysiological processes mediating cardiovascular diseases $(26,27)$, we hypothesized that the oxysterols levels can be altered in plasma of AAA patients. The clinical significance of circulating $7-\mathrm{KC}$ in the setting of AAA and its relationship with the induction of ER stress in the vascular wall has not yet been evaluated.

The purpose of the present study is to elucidate the implication of ER stress and its deleterious relationship with mitochondria in AAA disease. Moreover, we analyzed circulating levels of several oxysterols in the plasma focusing on 7-KC. Our findings demonstrate the enhanced expression of ER stress markers and alterations in mitochondrial biogenesis, autophagy and in the oxysterols profile of AAA patients, and suggest that therapeutic approaches aiming to reduce ER stress and to increase mitochondrial biogenesis could be useful to manage the progression of AAA disease.

\section{MATERIALS AND METHODS}

\section{Human samples}

Human aneurysmatic and blood samples were obtained from patients undergoing open repair for AAA at the Hospital de la Santa Creu i Sant Pau (HSCSP; Barcelona, Spain), while healthy aortas were obtained from multi-organ donors. Approval to use the discarded human tissue was given by the Ethics Committee of

the HSCSP. Research has been carried out in accordance with the Declaration of Helsinki. Participation in the study of patients and control subjects was based upon informed consent of patients or legal representatives. 
Abdominal aorta segments were obtained from patients $(n=94)$ and control subjects $(\mathrm{n}=17)$, following strict standard operating procedures and ethical guidelines. Samples of control subjects had no post-mortem evidence of AAA. Samples were rapidly collected and stored at $-80{ }^{\circ} \mathrm{C}$ for subsequent RNA and protein studies or processed for immunohistochemical analysis.

The blood samples from healthy donors included in this study were provided by the Barcelona Tissue Bank and accomplished the following inclusion criteria: healthy subjects aged 55 to 65 years, without type 2 diabetes, non-hypertensive (or pharmacologically-controlled hypertension individuals) and without any cardiovascular complication.

\section{Cell culture}

Human VSMC were isolated from abdominal aorta of multi-organ donors by an explant procedure as previously described (28). Briefly, endothelium denuded medial tissue was cut into $2-4 \mathrm{~mm}$ cubes that were transferred to a $25 \mathrm{~cm}^{2}$ culture flask containing $5 \mathrm{~mL}$ of pre-warmed culture medium M199 (Gibco, Carlsbad, CA, USA) supplemented with $10 \%$ foetal calf serum (FCS; Biological Industries, Kibbutz Beit-Haemek, Israel) and antibiotics (100 U/ml penicillin and $0.1 \mathrm{mg} / \mathrm{ml}$ streptomycin). VSMC migrate out from the explants within 2-3 weeks. Then, after removing the explants from the flask surface, cells were trypsinised, used as P1 stage cells, and routinely subcultured. Human VSMC were cultured in M199 supplemented with $20 \%$ FCS, $2 \%$ human serum, $2 \mathrm{mmol} / \mathrm{L}$ L-glutamine (Invitrogen) and antibiotics.

For experimental procedures, cells between passages 3 to 6 were seeded in multiwell plates and subconfluent cells were starved in medium supplemented with 
$1 \%$ FCS for $24 \mathrm{~h}$ prior to the addition of $15-30 \mu \mathrm{M}$ of $7-\mathrm{KC}$ (Sigma Aldrich) or vehicle (ethanol; $0.1 \%$ for $24-48 \mathrm{~h}$ in the presence or absence of human Angiotensin II $\left(10^{-7} \mathrm{M}\right)$ for $48 \mathrm{~h}$ (Sigma Aldrich). In another set of experiments, VSMC were pre-incubated with two antioxidants, Tempol $(10 \mu \mathrm{M})$ or $\mathrm{N}$-acetylcysteine (NAC, $10 \mathrm{mM}$ ), $1 \mathrm{~h}$ prior to exposure to 7-KC for $24 \mathrm{~h}$.

\section{Assessment of apoptosis in VSMC.}

Viable, apoptotic and necrotic cells were evaluated by using Annexin V FITC Apoptosis Detection Kit I with 7-AAD (BD, Pharmingen). Briefly, treated cells $\left(1 \times 10^{6} / \mathrm{mL}\right)$ were resuspended in binding buffer $(1 \times)$ and stained with Annexin V FITC and 7AAD according to the manufacturer's instructions. Cytometric experiments were carried out with a Beckman Coulter's Epics XL flow cytometer (Beckman Coulter, Brea, CA) equipped with a 488-nm argon laser. For each sample, at least 10,000 events were acquired. Samples incubated with the vehicle were used as a negative control. Data were analysed with the Expo32 software (Beckman Coulter, Brea, CA).

\section{Total mRNA and protein isolation from tissue and cells.}

The RNeasy Fibrous Mini Kit (Qiagen, Venlo, Netherlands) was used to isolate total RNA from human aortic samples following the manufacturer's recommendation. Total RNA isolation from human VSMC was performed using the Tripure reagent (Roche Diagnostics; Indianapolis, IN, USA) following the manufacturer's instructions. RNA integrity was determined by electrophoresis in agarose gels and was quantified by a NanoDrop 1000 Spectrophotometer (Thermo Scientific). Protein lysates from human aortic samples were prepared in a RIPA buffer $(150 \mathrm{mM} \mathrm{NaCl}, 1 \%(\mathrm{v} / \mathrm{v})$ Triton X-100, 0.5\% (w/v) sodium deoxycholate, 
$0.1 \%(\mathrm{w} / \mathrm{v}) \mathrm{SDS}, 2 \mathrm{mM}$ EDTA, $50 \mathrm{mM}$ Tris- $\mathrm{HCl} \mathrm{pH}$ 8) by using a tissue homogenizer and following a standard protocol.

\section{Quantitative Real-Time PCR.}

DNase I-treated total RNA $(1 \mu \mathrm{g})$ was reverse transcribed into cDNA using the High Capacity cDNA Archive Kit (Applied Biosystems, Foster City, CA, USA) with random hexamers. Quantification of mRNA levels was performed by real-time PCR using specific primers and probes provided by the Assay-on-Demand system (Applied Biosystems) for human ATF6 (Hs00232586_m1), CHOP or DDIT3 (Hs99999172_m1), XBP-1 (Hs00231936_m1), heat shock protein 5 (HSPA5) or GRP78 (Hs99999174_m1), IRE1 or ERN1 (Hs00176385_m1), ATF4 (Hs00909569_g1), Suppressor/Enhancer of Lin-12-like (SEL1L; Hs01071406_m1), cysteine-rich with EGF-like domains 2 (CRELD2; Hs00360923_g1), mitochondrially encoded cytochrome c oxidase III (MT-CO3; Hs02596866_g1), CYBB (NOX2, Hs00166163_m1), CYBA (p22phox, Hs00609145_m1), NOX4 (Hs01379108_m1), nuclear factor erythroid 2-related factor 2 (NRF2 or NFE2L2; Hs00975961_g1), metalloproteinase 2 (MMP2) (Hs01548727_m1) and IL1 $\beta$ (Hs01555410_m1). Human peroxisome profilerator-activated receptor coactivator $1 \alpha(\mathrm{PGC} 1 \alpha)$, nuclear respiratory factor 1 (NRF1), mitochondrial transcription factor A (TFAM) and Cytochrome B (Cyt B) mRNA was determined by using real-time PCR with SYBR Green PCR Master mix (Applied Biosystems, Thermo Fisher Scientific Inc, Waltham, MA, USA) and the following primers (www.Biomers.net, Donau, Germany): Cyt B forward 5'-AGACAGTCCCACCCTCACAC-3' and reverse 5'-AAGAGAAGTAAGCCGAGGGC-3'; 5'-CCTGTGGATGAAGACGGATT-3' and reverse 5'-TAGCTGAGTGTTGGCTGGTG-3'; TFAM forward 5'-GATGCTTATAGGGCGGAG-3' and reverse 5'-GCTGAACGAGGTCTTTTTGG-3'; NRF1 forward 5'-GATCGTCTTGTCTGGGGAAA-3' and reverse 5'-GGTGACTGCGCTGTCTGATA-3'; and COX1 forward 5'GGCCTGACTGGCATTGTATT-3' and reverse 5' TGGCGTAGGTTTGGTCTAGG-3' as previously described (28). In order to appropriately apply this method, we checked that the target genes were amplified 
with comparable efficiencies $(>95 \%$, calculated on the basis of the slope of calibration curves). As endogenous controls glyceraldehyde 3-phosphate dehydrogenase (GAPDH; Hs02758991_g1) and $\beta$-actin (Hs99999903_m1) were used. Each sample was amplified in duplicate. Similar results were obtained after normalisation to either housekeeping gene. Quantitative RT-PCR was carried out in an ABI PRISM 7900HT Sequence Detection System (Applied Biosystems). Relative mRNA levels were determined using the $2^{-\Delta \Delta C t}$ method.

\section{Immunostaining and histology.}

Aorta samples were fixed in 4\% paraformaldehyde/0.1 M PBS (pH 7.4) for $24 \mathrm{~h}$ and embedded in paraffin or transferred to a cryomold containing OCT embedding medium (Tissue Tek, Sakura, Leiden, NL) and snap-frozen in liquid nitrogen. The histological characterization of aortic samples was performed by Masson's trichrome and by orcein stain. Immunostaining assays were performed in aortic sections from human AAA and donor samples as previously described (29).

Briefly, for immunohistochemistry assays aortic sections $(5 \mu \mathrm{m})$ were deparaffinized in xylene, rehydrated in graded ethanol, and treated with $0.3 \%$ hydrogen peroxide for 30 min to block peroxidase activity. Then, samples were blocked with $10 \%$ of normal serum and incubated with antibodies against $\mathrm{CHOP}$, cleaved-caspase 3 (9661, Cell Signaling; Boston, MA, USA) and CD68 (M0876, Dako) overnight at $4{ }^{\circ} \mathrm{C}$. After washing, samples were incubated for $1 \mathrm{~h}$ with a biotinylated secondary antibody (Vector Laboratories, Peterborough, UK). After rinsing 3 times in PBS, standard Vectastain ( $\mathrm{ABC}$ ) avidin-biotin peroxidase complex (Vector Laboratories) was applied, and the slides were incubated for 30 min. Colour was developed using 3,3'-diaminobenzidine (DAB) and sections were counterstained with haematoxylin before dehydration, clearing, and mounting. Negative controls, in which the primary antibody was omitted, were included to test for non-specific binding. Results were quantified and expressed as percentage of 
positive area versus total area or as positive cell number per area in independent sections of AAA for CHOP, cleaved-caspase 3 and CD68.

For antigen colocalization studies, double-fluorescence immunostaining was performed using a sequential method. After deparaffinization, antigen retrieval and permeabilization with PBS-0.1\% Triton X-100, sections were blocked with PBS containing 5\% albumin for $1 \mathrm{~h}$ at room temperature. Incubation with mouse polyclonal antibodies against $\mathrm{ATF} 6, \mathrm{XBP}-1$ or $\mathrm{CHOP}\left(4^{\circ} \mathrm{C}\right.$ overnight $)$, and a rabbit polyclonal antibody against smooth muscle $\alpha$-actin (ab5694, Abcam) or CD3 (SK202, Dako), were then sequentially applied. After washing the primary antibodies, donkey anti-mouse IgG conjugated to Alexa Fluor 594, and chicken anti-rabbit IgG conjugated to Alexa Fluor 488 (Molecular Probes, Life Technologies) were applied for $1 \mathrm{~h}$ at room temperature. Negative controls, in which the primary antibody was omitted, were also included. Samples were then mounted with ProLong Gold antifade reagent with DAPI (Molecular Probes, Life Technologies Co., Eugene, OR). Images were obtained using an SP5 Leica confocal microscope.

\section{Western blot.}

Tissue lysates were separated by SDS-PAGE and transferred to $0.45 \mu \mathrm{m}$ polyvinylidene difluoride membranes (Immobilon, Millipore. Merck KGaA, Darmstadt, Germany). Blots were incubated with antibodies directed against ATF6 (NBP1-40256), XBP-1 (NBP1-75514), CHOP (NB600-1335), dynamin related protein 1 (DRP-1; NB110-55288) and p62 (NBP1-48320) purchased from Novus Biologicals (Bio-Techne LD-R\&D Systems Europe Ltd, Abingdon, UK); rabbit polyclonal antibodies against Beclin 1 (PA1-16857) and LC3A/LC3B (PA1-16931) 
were obtained from Pierce (ThermoFisher Scientific). Equal loading of protein in each lane was verified by $\beta$-actin (A5441, Sigma Aldrich).

\section{TUNEL assay}

Recombinant Terminal Deoxynucleotidyl transferase (rTdT) mediated nick-end labeling (TUNEL) was performed using the Dead End Fluorometric Tunel System (Promega) according to the manufacturer's guidelines. Four micrometer paraffin sections from AAA and donor aortas were deparaffinized, fixed in methanol-free paraformaldehyde before and after proteinase $\mathrm{K}$ treatment at $20 \mu \mathrm{g} / \mathrm{ml}$ for $8-10$ min at room temperature. The sections were incubated with the nucleotide mixture (which included fluorescein-tagged dUTP) and rTdT enzyme for an hour at $37^{\circ} \mathrm{C}$. The slides were mounted using SlowFade Gold antifade reagent with DAPI (Molecular Probes, Invitrogen) and immunofluorescent signals were viewed using a fluorescence microscope (Nikon Eclipse 55i).

\section{Detection of superoxide anion production in situ}

The oxidative fluorescent dye dihydroethidium (DHE; Molecular Probes ${ }^{\mathrm{TM}}$ Invitrogen, Carlsbad, CA, USA) and the fluorogenic dye MitoSOX ${ }^{\mathrm{TM}}$ Red were used to evaluate superoxide anion $\left(\mathrm{O}_{2}{ }^{--}\right)$production in frozen aorta sections preserved in OCT. The fluorescent dye MitoTracker ${ }^{\mathrm{TM}}$ Green FM (Molecular Probes, Thermo Fisher Scientific) was used to stain mitochondria.

Briefly, frozen tissue embedded in OCT were cut into $10 \mu \mathrm{m}$ thick sections, placed on a glass slide and equilibrated under identical conditions for $30 \mathrm{~min}$ at $37^{\circ} \mathrm{C}$ in Krebs-HEPES buffer (in mM: $130 \mathrm{NaCl}, 5.6 \mathrm{KCl}, 2 \mathrm{CaCl}_{2}, 0.24 \mathrm{MgCl}_{2}, 8.3$ HEPES, 11 glucose, $\mathrm{pH}=7.4)$. Fresh buffer containing DHE $\left(5 \times 10^{-6} \mathrm{M}\right)$ was applied topically onto each tissue section, cover-slipped and incubated for $30 \mathrm{~min}$ in a light-protected humidified chamber at $37{ }^{\circ} \mathrm{C}$. For MitoSox red and 
Mitotracker green stainings, a solution of $1 \times 10^{-6} \mathrm{M}$ of each dye in phosphate buffer was applied onto the tissue sections and incubated for $15 \mathrm{~min}$ in a light-protected humidified chamber at $37^{\circ} \mathrm{C}$. Slides were subsequently washed with phosphate buffer to remove the excess of dyes and mounted using SlowFade Gold antifade reagent (Molecular Probes, Invitrogen).

Aorta images were viewed by fluorescent laser scanning microscope (20X objective in a Leica DMI 3000 microscope; Leica AG, Germany) and quantified using the Leica LAS, 4.3 analysis system (Leica AG, Germany). Histological sections of aortas from 10 different patients and different fields in each section per sample were quantified, averaged and expressed as relative fluorescent units (RFU).

\section{Determination of oxysterols in plasma}

Plasma was separated from EDTA blood collection tubes within 2 hours of the blood draw. Plasma was frozen in aliquots at $-80^{\circ} \mathrm{C}$ promptly after separation. Absolute quantification of oxysterols (24-hydroxycholesterol (HC); 25-HC; 27-HC; $7 \alpha-\mathrm{HC} ; 7 \beta-\mathrm{HC}$ and $7-\mathrm{KC}$ was performed by liquid chromatography coupled to tandem mass spectrometry (LC-MS/MS) with atmospheric pressure chemical ionization (APCI) interface (UHPLC-(+)APCI-MS/MS) in the Centre for Omic Sciences (Reus, Tarragona, Spain as previously described $(30,31)$.

Briefly, two extraction methods were developed and validated in plasma samples: one to quantify free oxysterols (7-KC and 27-HC) based on Helmschrodt et al (30) containing the internal standards deuterated and the other to quantify total oxysterols introducing a saponification step based on Mukhopadhyay et al and Narayanaswamy et al (31,S1). Simultaneously, a development and validation of LC-(APCI)-MS/MS methodologies for each of the compounds was carried out. An 
UHPLC 1290 Infinity II Series coupled to a QqQ/MS 6490 Series, both Agilent Technologies (Agilent Technologies, Sta. Clara, CA, USA) was used to analyze the extracts. $25-\mathrm{HC}$ was found $<\mathrm{MQL}$ (ranged from 1 to $5 \mathrm{ng} / \mathrm{mL}$ ) in all the samples.

Analytical standards 7- $\beta-\mathrm{HC}, 25-\mathrm{HC}$ and $7-\mathrm{KC}$ were obtained from Sigma Aldrich. 24-S-HC and 27-HC from Enzo life Sciences and 7-KC-d7 from Toronto Research Chemicals.

\section{Determination of the lipid profile in plasma}

The lipid profile was determined enzymatically using commercial kits adapted to a COBAS 6000 autoanalyzer (Roche Diagnostics, Bassel, Switzerland). The lipid profile included total cholesterol, triglycerides, HDL-C, LDL cholesterol and VLDL cholesterol. LDL and VLDL cholesterol were calculated with the Friedewald's equation, except when TG were higher than $3 \mathrm{mmol} / \mathrm{L}$, where VLDL was separated by ultracentrifugation according to the National Cholesterol Education Program (NCEP) recommendations (32).

\section{Statistical analysis}

GraphPad Prism 4.0 software (GraphPad, USA) was used for statistical analysis. Data were expressed as mean \pm SEM and values of $p \leq 0.05$ were considered significant. When data fitted a normal distribution differences between two groups were assessed using the Student's t test (two-tailed) and one-way ANOVA and the Bonferroni test for more than two groups. When normality failed, we used the Mann-Whitney rank sum test to compare two groups and Kruskal-Wallis one-way analysis of variance on ranks for multiple comparisons (Dunn's method). Pearson product-moment correlation coefficient was used to determine the association between variables. Multivariate logistic regression analysis using the 
statistical software package $\mathrm{R}$ (www.r-project.org) was performed to assess potential confounding factors. The results were expressed as odd ratio (OR) and $95 \%$ confidence intervals $(\mathrm{CI})$.

\section{RESULTS}

\section{ER stress is present in AAA}

The histological examination of abdominal aorta samples from AAA patients and donors showed the disturbance in overall abdominal aorta wall morphology and the extracellular matrix (ECM) disorganization frequently observed in AAA (Fig. S1A-B). Table 1 shows demographic and clinical data of patients and donors included in this study.

The expression of ER stress markers that represent the three pathways of the UPR was analyzed in abdominal aorta samples from AAA patients $(n=96)$ and healthy donors (HD, $\mathrm{n}=17)$. IRE1, XBP-1, ATF6, and CHOP mRNA levels were significantly higher in AAA, however, no differences were observed for ATF4 and HSPA5 in comparison with aortas from donors (Fig. 1A-F). After adjusting for age, sex, smoking, and comorbidities (hypertension and type 2 diabetes), the differences between AAA patients and donors remained statistically significant except for CHOP expression (table S1).

Additionally, we studied the expression of two recently discovered ER stress-inducible genes: CRELD2 and SEL1L (Fig. 1G-H). Our results showed higher mRNA levels of CRELD2 (Fig. 1G) and SEL1L (Fig. 1H) in AAA, indicating an activation of the endoplasmic reticulum-associated degradation (ERAD) process.

The higher expression of XBP-1, ATF6, and CHOP in aneurysmatic samples was confirmed by Western blot (Fig. 2A-C) and was further assessed by 
immunofluorescence. A strong immunostaining for ATF6, XBP-1, and CHOP was observed in the media layer of aneurysmatic samples, specifically in VSMC (Fig. 2D). Double-staining of ER stress markers with CD3 revealed that only ATF6 and XBP-1 co-localized in lymphocytes in areas of inflammatory infiltrates (Fig. 2D). As a pro-apoptotic effector of the UPR, CHOP expression in VSMC from AAA was associated with a higher expression of cleaved caspase 3. Additionally, an enhanced presence of apoptotic cells was detected in aneurysmatic wall assessed by TUNEL assay (Fig. S2A-C).

\section{Oxidative stress is higher in the aortic wall of human AAA than in healthy}

\section{abdominal aorta.}

In aneurysmatic abdominal aortas the expression of members of the nicotinamide adenine dinucleotide phosphate-oxidase (NADPH oxidase) family was altered. Specifically, NOX2 and p22phox mRNA levels were higher, while NOX4 expression was lower compared with donor samples. The expression of NRF2 was not altered in AAA patients (Fig. 3A-D). Superoxide anion production measured by DHE staining was greater in AAA compared with donors (Fig. 3E). Because mitochondria are a target of superoxide anion produced by NADPH oxidase, we stained abdominal aortic samples with Mitosox red probe. As observed in Fig. 3F an increase in mitochondrial ROS was also detected in AAA patients. The staining of mitochondrial mass by using the fluorescent probe Mitotracker green revealed that mitochondria abundance (green fluorescence detected between the autofluorescent elastic lamellae; please see negative controls) was lower in aneurysmastic samples that in the arterial wall from donors (Figure 3G). Further, inmunostaining analysis revealed a partial co-localization of superoxide anion 
(DHE staining) with XBP-1, ATF6 and CHOP in frozen aortic samples of AAA patients (Fig. S4).

\section{Mitochondrial biogenesis and mitophagy are disturbed in AAA.}

To analyze mitochondrial biogenesis we determined the ratio of the expression of Cytochrome $\mathrm{B}(\mathrm{CytB})$ and cytochrome $\mathrm{C}$ oxidase $(\mathrm{CytC})$ vs. that of $\beta$-actin as indicator of mitochondrial biogenesis. These ratios were significantly reduced in AAA patients compared to donors supporting a decrease of mitochondrial biogenesis (Fig. 4A-B). As expected, the mRNA levels of PGC1 1 , a major regulator of the mitochondrial biogenesis, and its downstream targets: NRF1 and mitochondrial TFAM, a direct regulator of mitochondrial DNA replication and transcription, were dramatically reduced in human AAA samples compared to donors (Fig. 4C-E).

Because mitochondrial autophagy (or mitophagy) plays an important role in mitochondrial turnover, we analyzed the expression of mitophagy and autophagy markers in tissue lysates of AAA patients and donors. The expression of DRP1 was lower while protein expression of LC3II, Beclin-1 and p62, three autophagy regulatory proteins, was higher in AAA compared to donors (Fig. 4F).

\section{The oxysterols profile is altered in the plasma of AAA patients}

We measured the levels of free 7-KC levels and other oxysterols (24S-HC, 25-HC, 27-HC, 7- $\beta-\mathrm{HC}$, and $7-\alpha-\mathrm{HC})$ in the plasma of AAA patients $(\mathrm{n}=94)$ and healthy donors $(\mathrm{n}=47)$ to determine their profile. We observed that 7-KC levels were significantly higher in aneurysmatic patients (Fig. 5A). However, 7- $\alpha-\mathrm{HC}, 24$ (S)-HC, and 27-HC (analyzed as free oxysterol and as a hydrolysed oxysterol 
extracted from plasma) were significantly lower in AAA patients compared to donors (Fig. 5C-E). 7- $\beta$-HC plasma levels were similar between donors and AAA (Fig. 5B), while 25-HC levels were undetectable. After adjusting for age and sex, the differences for $7-\mathrm{KC}, 24-\mathrm{S}-\mathrm{HC}$ and $7 \alpha-\mathrm{HC}$ plasma levels between AAA patients and donors remained statistically significant (table S2).

We then studied possible correlations between levels of the different oxysterols with total cholesterol (TC), HDL-cholesterol (HDL-C), LDL-cholesterol (LDL-C), VLDL-cholesterol (VLDL-C) and triglycerides (TG) levels in the plasma of patients and donors and the results are summarized in table 2. A positive correlation was observed between $24 \mathrm{~S}-\mathrm{HC}$ or $27-\mathrm{HC}$ levels and TC and LDL-C levels. Furthermore, only 24(S)-HC levels correlated with TC and LDL-C in either donors or AAA patients. LDL-C levels positively correlated with 27-HC levels in the AAA group but not in donors. In AAA patients HDL-C levels were significantly associated with $24 \mathrm{~S}-\mathrm{HC}$ and $7 \alpha-\mathrm{HC}$ levels but they did not correlate in donors. A positive correlation was also found between $24(\mathrm{~S})-\mathrm{HC}$ or $27-\mathrm{HC}$ levels and TG and VLDL-C levels in AAA patients but not in donors. None of the lipid profile variables were significantly associated with 7-KC levels in either AAA patients or donors. No associations were found between values of abdominal aortic diameter and oxysterols levels in AAA patients (table 2). Interestingly, in AAA patients, 7-KC plasma levels positively correlated with the aortic expression of IRE1, ATF6 and CHOP (Fig. 5F-H). Further associations of 7-KC levels were established with the local expression of NAPDH oxidase subunits in AAA (Fig. $5 \mathrm{I}-\mathrm{J})$.

ER stress activation and mitochondrial biogenesis alteration in human VSMC 


\section{after exposure to $7-\mathrm{KC}$.}

To investigate the effects of 7-KC on vascular cells, human VSMCs were incubated with $15 \mu \mathrm{M}$ of 7-KC, concentration established on the basis of preliminary studies revealing that primary VSMC are very sensitive to 7-KC-induced apoptosis at a higher dose (data not shown). The percentages of early apoptotic, late apoptotic cells, necrotic cells, and live cells in 7-KC-induced cells were quantified by flow cytometry (Fig. S5).

The expression of the ER stress markers ATF4, ATF6, CHOP, HSPA5, IRE1, SEL1L and CREDL2 was significantly induced by 7-KC in human VSMC. When cells were co-incubated with Ang II, as a vasoactive peptide and an important mediator of the aneurysmatic process, we discovered that Ang II triggered an additional increment in CHOP mRNA levels which suggests a synergistic effect (Fig. 6A-G).

Additionally, we studied whether mitochondrial biogenesis biomarkers, MMP2 and the pro-inflammatory marker IL-1 $\beta$ were altered in VSMCs treated with 7-KC and co-incubated or not with Ang II. We observed that 7-KC reduced PGC1 $\alpha$ and TFAM expression, while mRNA levels of NRF1 were not significantly altered (data not shown) (Fig. 6H-I). Furthermore, MMP2 and IL-1 $\beta$ mRNA levels were up-regulated after exposure to 7-KC (Fig. 6J-K). The co-incubation with Ang II did not either change the effects of $7-\mathrm{KC}$ on mitobiogenesis markers or in IL-1 $\beta$ expression but triggered a further increment in MMP2 expression (Fig. 6H-K).

Because it has been well-established that 7-KC triggers oxidative stress in vascular cells $(33,34)$, we determined whether ROS production contributes to 7KC-mediated responses. The preincubation of VSMC with the antioxidants 
Tempol and/or NAC attenuated the 7-KC-mediated induction of several ER stress and oxidative stress markers expression (Fig. S6).

\section{DISCUSSION}

Currently, therapeutic strategies in AAA are restricted to invasive surgical repair for those AAA patients with a high risk of rupture. No effective pharmacological strategies are yet established to suppress the development of AAA and to prevent the need for invasive aneurysm repair $(5-9,35)$. Therefore, the search for new therapeutic targets to limit AAA progression is a challenge that requires further research.

In the last decade, an increasing number of studies have reported that an exacerbated induction of ER stress and mitochondrial stress could be detrimental in multiple pathologies including cardiovascular diseases. Emerging evidence for protein trafficking dysregulation and ER abnormalities compatible with ER stress have been described in cardiovascular pathologies (36-38). Nevertheless, ER stress has not been considered as a therapeutic target in AAA and little is known about its

role in the vascular wall degeneration that occurs during AAA formation, expansion, and rupture. Our results show evidence of the involvement of ER stress and its deleterious cross-talk with mitochondria, oxidative stress generation and apoptosis in human AAA.

In human aneurysmatic samples from our patient cohort, the UPR response was activated as demonstrated by the higher expression of IRE1-XBP1 axis, ATF6, and CHOP compared with abdominal aortas from donors, while no differences between were observed for ATF4 and HSPA5, indicating a differential activation 
of the three axis of the ER stress response at the end stage of AAA. To reinforce our data, we studied the expression of two recently reported players involved in ER stress known as CRELD2 and SEL1L. Interestingly, their expression was higher in aneurysmatic samples. CRELD2 is implicated in the processing and trafficking of proteins through the ER-Golgi apparatus and it is up-regulated by ATF6 (39) while SEL1L is involved in ERAD (40) and targets misfolded secretory and membrane proteins in the ER for proteasomal degradation (41). Therefore, their induction is consistent with the enhanced expression of ATF6 detected in aneurysmatic patients.

According to the immunofluorescence studies, the induction of ER stress markers was observed mainly in VSMC and to a lesser extent in the inflammatory infiltrate of the aneurysmatic wall. These results suggest that ER stress could be related to arterial wall degeneration occurring during AAA progression. Recently, it has been reported that inhibition of ER stress with Tauroursodeoxycholic acid (Tudca), with intermedin 1-53 peptide or by the treatment with statins reduced the incidence of AAA in a murine model, supporting the role of ER stress in AAA formation (42-44). However, to our knowledge, this is the unique study that has exhaustively analyzed the three effector branches of the ER stress response in a big cohort of AAA patients.

We found that the profile of oxysterols was clearly altered in our cohort of aneurysmatic patients. Interestingly, a similar oxysterol profile was recently reported in a study performed with patients suffering from multiple sclerosis (MS) (31). Other studies have shown that oxysterols levels are altered not only in MS but also in other neurodegenerative diseases such as Alzheimer's and Parkinson's disease, in which ER stress seems to play a critical role $(31,45,46) .7-\mathrm{KC}$ levels 
were significantly higher in plasma of AAA patients compared with healthy donors and more interestingly, our data show a positive correlation between 7-KC plasma levels and the vascular expression of ER stress markers in AAA. In this context, recent publications emphasize on the importance of circulating oxysterols in the development of cardiovascular diseases (CVD), and in particular the augmentation of 7-KC levels has been associated with CVD events $(26,27)$. In support to our results, other authors previously reported that cigarette smoking increases plasmatic 7-KC levels in humans (47). Since smoking is considered one of the main risks factors for the development of AAA disease, an imbalance in the oxysterols levels could be considered as a causative factor. However, the pathophysiological repercussion of the higher 7-KC levels in aneurysmatic patients remains to be defined.

In human VSMC, 7-KC evoked the induction of ER stress and apoptotis as reported before by Pedruzzi E et al (48). It has been described that 7-KC amplifies inflammatory processes and regulates the expression of inflammatory cytokines and MMPs (21). Accordingly, we found an induction of MMP-2 and IL-1 $\beta$ expression in human VSMC triggered with 7-KC supporting the deleterious effect that this oxysterol could trigger in the vascular wall during AAA disease. In our cohort of patients, the levels of $7 \beta-\mathrm{HC}$ remained unchanged compared to donors. In contrast, $7 \alpha-\mathrm{HC}, 24(\mathrm{~S})-\mathrm{HC}$ and $27-\mathrm{HC}$ levels were significantly lower in AAA. Compared to other oxysterols, 24-HC levels are found to be evidently dependent upon age and they are strikingly decreased in the sixth decade of life. A decrease in 24-HC levels has been reported before in MS and Alzheimer's patients (49). However, no correlations of either oxysterols levels and the age of individuals were found, which is anticipated due to the advanced age of our cohort of AAA 
patients and donors. 24-HC and 27-HC are ligands of liver X receptors (LXRs) which controls the transcription of genes involved in cholesterol homeostasis and innate immunity (50). We hypothesize that a decrease in 24-HC, 27-HC, and $7 \alpha-\mathrm{HC}$ levels could disrupt cholesterol homeostasis and exacerbate an immunological response in the vascular wall through a reduction in LXRs activation. Altogether, these findings suggest that a disruption in the oxysterols network could contribute to AAA disease progression.

Increased circulating 7-KC could entail the unique intersection of enhanced intracellular free cholesterol and increased oxidative stress. Among the oxysterols, 7-KC and 27-HC, both induce ROS in VSMC and non vascular cells (51-53). Interestingly, we observed that the pre-incubation of VSMC with antioxidants attenuated the increased expression of oxidative and ER stress markers induced by 7-KC. In this context, we noticed a positive correlation between NOX2 or p22phox expression and 7-KC levels in AAA patients, supporting the negative impact of this oxysterol on vascular homeostasis. Oxidative stress is closely related to ER stress induction and it is also known to be involved in the pathological mechanisms underlying human aortic abdominal aneurysm (54). ER stress up-regulate NADPH oxidase expression and activity, the major source of vascular ROS (12). In turn, $\mathrm{O}_{2}^{-}$ modulates inflammatory reactions and can enhance leukocyte recruitment, which also contribute to AAA progression (55). In our cohort of AAA patients, we observed an increased expression in NOX2 and p22phox subunits of NADPH oxidase and an exacerbated production of superoxide anion and mitochondrial reactive oxygen species (ROS) in aneurysmatic tissue. In contrast, NOX4 expression was lower in AAA aorta than in donor samples as previously described by Guzik et al in a small cohort of AAA patients (56). 
Moreover, ER stress is associated with mitochondrial dysfunction by promoting mitochondrial ROS generation and apoptosis (19). Mitochondria-dependent ROS/apoptosis activation plays a critical role in the progression of AAA in animal models of the disease (57). It has been shown that induction of mitochondrial biogenesis leads to a decrease in apoptosis and ROS production. Conversely, mitochondrial biogenesis is down-regulated by ROS $(58,59)$. In our cohort of AAA patients we observed that the expression of transcription factors that activate mitochondrial biogenesis and regulate mitochondrial function (PGC1 $\alpha, \mathrm{NRF} 1$, and TFAM) as well as Cyt B and Cyt C oxidase were all decreased in aneurysmatic tissue, suggesting a reduction of mitochondrial content. Consistent with this, a previous study enrolling a limited number of patients reported a decrease in PGC1 $\alpha$ in the medial wall of aneurysmatic aorta (60). Moreover, Mitotracker staining evidenced a reduction in the mitochondrial mass in aneurismatic wall. Therefore, our results reinforce the concept that the maintenance of mitochondrial homeostasis could be an interesting therapeutic strategy in AAA and support that pharmacological approaches increasing mitochondrial biogenesis could ameliorate vascular degeneration.

Activation of ER stress can trigger changes not only in mitochondrial function but also in autophagy (61) and 7-KC induces autophagy and apoptosis in advanced atherosclerotic plaques (23). Autophagy is a major catabolic process that delivers proteins, cytoplasmic components, and organelles to lysosomes for degradation and recycling. While autophagy is a critical cytoprotective mechanism, it has been suggested to also lead to cellular death depending on cell circumstances $(17,62)$. In this study, we found a higher expression of Beclin-1, LC3II and p62 in human aneurysmatic aortas. In turn, mitophagy eliminates the accumulation of damaged 
mitochondria that otherwise result in excessive ROS production and apoptosis $(61,63)$. Protein levels of DRP1, a marker for mitophagy, was lower in AAA than in donors. This reduction could be derived from the decrease in mitochondrial content, but could also suggest that this protective mechanism was no longer active in the aneurysmatic wall.

We found the inherent restrictions of the work with human specimens. The availability of aortic specimens is hampered by the requirement of multiorganic donors, from which it is almost impossible to obtain blood samples. Therefore, healthy aorta and blood donors are not from the same group of individuals. Further, on the basis of our data, we could not definitively demonstrate a direct role of ER stress in human AAA and we can only speculate that ER stress is related to AAA development as part of the pathophysiological process. However, it should be noted, that inhibition of ER stress limits AAA development in animal models, supporting the contribution of ER stress to this disease $(42-44,64)$. Finally, there are important differences between AAA patients and healthy donors regarding clinical data. Full clinical information and pharmacological treatments that might interfere in our study are lacking for blood donors. Nevertheless, after performing the corresponding statistical analysis we could exclude age, sex, smoking, hypertension and type 2 diabetes as confounding factors of our current observations. In summary, our study highlights the importance of ER stress and mitochondrial biogenesis in AAA and demonstrates, for the first time, the associated disturbance of the oxysterols profile in this disease. Our data supports that the increase in 7-KC levels and the decrease in plasma concentrations of several hydroxycholesterols could contribute to ER stress activation and chronic inflammation in the aorta of AAA patients. Most importantly, the present study 
suggests that ER stress and mitochondrial dysfunction could be potential targets for novel therapeutic strategies to limit aneurysm progression in patients who are diagnosed at an early stage of AAA in screening programs.

\section{CLINICAL PERSPECTIVES}

- The surgical treatment of AAA is only recommended for aneurysms that are greater than $5.5 \mathrm{~cm}$ in diameter. Moreover, for those AAAs which are first diagnosed when their diameter is less than $5.5 \mathrm{~cm}$, pharmacologic treatment could be beneficial in slowing or reducing AAA expansion and rupture. Numerous pharmacologic interventions have been proposed to limit AAA growth and rupture but the negative results obtained with these therapies evidence the need of new pharmacological approaches to manage this disease.

- The results of the present study support an important role of ER stress activation and reduction of mitochondrial biogenesis in AAA and indicate a strong association between an enhanced expression of ER stress markers, oxidative stress and 7-KC in AAA.

- Our findings suggest that ER stress and mitochondrial biogenesis could be potential therapeutic targets to limit AAA progression.

\section{ACKNOWLEDGMENTS}

Authors are grateful to Sonia Alcolea for her technical assistance; to Dr. Nuria Canela for her technical support at the COS center (Reus, Tarragona) and to Dr. David de Gonzalo-Calvo for his assistance with the statistical analysis using the software package R.

\section{DECLARATION OF INTERESTS}


No competing interests declared.

\section{FUNDING}

This work was supported by the Spanish Ministerio de Economía y Competitividad (MINECO)-Instituto de Salud Carlos III (ISCIII) [grants CP15/00126 and PI17/08137 to MG and PI18/0919 to CR] and RTI2018-094727-B-100 to JMG and by CIBERCV (CB16/11/00257). The study was co-founded by Fondo Europeo de Desarrollo Regional (FEDER)-The way to build Europe. MNM and MG are supported by funds provided by ISCIII (grant CP15/00126, Miguel Servet I program) and LC is a recipient of a FI fellowship AGAUR.

\section{AUTHOR CONTRIBUTION}

MG conceived, designed and supervised the study. MNM and MG performed experiments, analysed and interpreted data. JF, JRE, MC and MG were responsible for the clinical aspects of the study; participated in patients material collection and analysis. CR, MK, LC and JMG conceived specific experiments, revised and carried out results interpretation. MG drafted the manuscript. All the authors revised the manuscript for important intellectual content and gave their final approval of the submitted version.

\section{REFERENCES}

1. Nordon, I.M., Hinchliffe, R.J., Loftus, I.M., Thompson, M.M. (2011) Pathophysiology and epidemiology of abdominal aortic aneurysms. Nat. Rev. Cardiol. 8, 92-102, https://doi.org/10.1038/nrcardio.2010.180

2. Moll, F.L., Powell, J.T., Fraedrich, G., Verzini, F., Haulon, S., Waltham, M., et al. (2011) Management of abdominal aortic aneurysms clinical practice guidelines 
of the European society for vascular surgery. Eur. J. Vasc. Endovasc. Surg. 41, Suppl 1, S1, https://doi.org/10.1016/j.ejvs.2010.09.011

3. Brady, A.R., Thompson, S.G., Fowkes, F.G., Greenhalgh, R.M., Powell, J.T. (2004) Abdominal aortic aneurysm expansion: risk factors and time intervals for surveillance. Circulation 110, 16-21, https://doi.org/10.1161/01.CIR.0000133279.07468.9F

4. Deaths, percent of total deaths, and death rates for the 15 leading causes of death in 5-year age groups, by race, and sex: United States, 2013. https://www.cdc.gov/nchs/data/dvs/LCWK1_2013.pdf

5. Ferguson, C.D., Buettner, P.G., Golledge, J. (2011) Regarding "a meta-analysis of clinical studies of statins for prevention of abdominal aortic aneurysm expansion". J. Vas. Surg. 53, 1452-1453, https://doi.org/10.1016/j.jvs.2010.08.089

6. Samson R. (2012) Can pharmacologic agents slow abdominal aortic aneurysm growth?. Semin. Vasc. Surg. 25, 25-28, https://doi.org/10.1053/j.semvascsurg.2012.03.004

7. Dodd, B.R., Spence, R.A. (2011) Doxycycline inhibition of abdominal aortic aneurysm growth: a systematic review of the literature. Curr. Vasc. Pharmacol. 9, 471-478, https://doi.org/10.2174/157016111796197288

8. Yoshimura, K., Nagasawa, A., Kudo, J., Onoda, M., Morikage, N., Furutani, A., et al. (2015) Inhibitory Effect of Statins on Inflammation-Related Pathways in Human Abdominal Aortic Aneurysm Tissue. Int. J. Mol. Sci. 16, 11213-11228, https://doi.org/10.3390/ijms160511213 
9. Salata K, Syed M, Hussain MA, Eikelboom R, de Mestral C, Verma S, Al-Omran M (2018). Renin-angiotensin system blockade does not attenuate abdominal aortic aneurysm growth, rupture rate, or perioperative mortality after elective repair. J Vasc Surg. 67, 629-636.e2. doi: 10.1016/j.jvs.2017.09.007.

10. Hotamisligil Go S. (2010) Endoplasmic reticulum stress and atherosclerosis. Nat. Med. 16, 396-399, https://dx.doi.org/10.1038\%2Fnm0410-396

11. Kassan, M., Galán, M., Partyka, M., Saifudeen, Z., Henrion, D., Trebak, M., Matrougui, K. (2012) Endoplasmic reticulum stress is involved in cardiac damage and vascular endothelial dysfunction in hypertensive mice. Arterioscler. Thromb. Vasc. Biol. 32, 1652-1661, https://dx.doi.org/10.1161\%2FATVBAHA.112.249318

12. Galán, M., Kassan, M., Kadowitz, P.J., Trebak, M., Belmadani, S., Matrougui, K. (2014) Mechanism of endoplasmic reticulum stress-induced vascular endothelial dysfunction. Biochim. Biophys. Acta. 1843, 1063-1075, https://dx.doi.org/10.1016\%2Fj.bbamcr.2014.02.009

13. Ron, D., Walter, P. (2007) Signal integration in the endoplasmic reticulum unfolded protein response. Nat. Rev. Mol. Cell. Biol. 8, 519-529, https://doi.org/10.1038/nrm2199

14. Ozcan, U., Yilmaz, E., Ozcan, L., Furuhashi, M., Vaillancourt, E., Smith, R.O., Görgün, C.Z., Hotamisligil, G.S. (2006) Chemical chaperones reduce ER stress and restore glucose homeostasis in a mouse model of type 2 diabetes. Science 313, 1137-1140, https://dx.doi.org/10.1126\%2Fscience.1128294 
15. Wang, S., Kaufman, R.J. (2012) The impact of the unfolded protein response on human disease. J. Cell Biol. 197, 857-867, https://dx.doi.org/10.1083\%2Fjcb.201110131

16. Myoishi, M., Hao, H., Minamino, T., Watanabe, K., Nishihira, K., Hatakeyama, K., et al. (2007) Increased endoplasmic reticulum stress in atherosclerotic plaques associated with acute coronary syndrome. Circulation 116, 1226-1233, https://doi.org/10.1161/CIRCULATIONAHA.106.682054

17. Deegan, S., Saveljeva, S., Gorman, A.M., Samali, A. (2013) Stress-induced self-cannibalism: on the regulation of autophagy by endoplasmic reticulum stress. Cell. Mol. Life. Sci. 70, 2425-2441, https://doi.org/10.1007/s00018-012-1173-4

18. B'Chir, W., Maurin, A.C., Carraro, V., Averous, J., Jousse, C., Muranishi, Y., Parry, L., Stepien, G., Fafournoux, P., Bruhat, A. (2013) The eIF2alpha/ATF4 pathway is essential for stress-induced autophagy gene expression. Nucleic. Acids. Res. 41, 7683-7699, https://doi.org/10.1093/nar/gkt563

19. Timmins, J.M., Ozcan, L., Seimon, T.A., Li, G., Malagelada, C., Backs, J., Backs, T., Bassel-Duby, R., Olson, E.N., Anderson, M.E., Tabas, I. (2009) Calcium/calmodulin-dependent protein kinase II links ER stress with Fas and mitochondrial apoptosis pathways. J. Clin. Invest. 119, 2925-2941, https://doi.org/10.1172/JCI38857

20. Guarente, L. (2008) Mitochondria-a nexus for aging, calorie restriction, and sirtuins?. Cell 132, 171-176, https://doi.org/10.1016/j.cell.2008.01.007 
21. Gargiulo, S., Gamba, P., Testa, G., Leonarduzzi, G., Poli, G. (2016) The role of oxysterols in vascular ageing. J. Physiol. 594, 2095-2113, https://doi.org/10.1113/JP271168

22. Hayden, J.M., Brachova, 1., Higgins, K., Obermiller, L., Sevanian, A., Khandrika, S., Reaven, P.D. (2002) Induction of monocyte differentiation and foam cell formation in vitro by 7-ketocholesterol. J. Lipid. Res. 43, 26-35.

23. Arca, M., Natoli, S., Micheletta, F., Riggi, S., Di Angelantonio, E., Montali, A., et al. (2007) Increased plasma levels of oxysterols, in vivo markers of oxidative stress, in patients with familial combined hyperlipidemia: reduction during atorvastatin and fenofibrate therapy. Free. Radic. Biol. Med. 42, 698-705, https://doi.org/10.1016/j.freeradbiomed.2006.12.013

24. Sanson, M., Auge, N., Vindis, C., Muller, C., Bando, Y., Thiers, J.C., et al. (2009) Oxidized low-density lipoproteins trigger endoplasmic reticulum stress in vascular cells: prevention by oxygen-regulated protein 150 expression. Circ. Res. 104, 328-336, https://doi.org/10.1161/CIRCRESAHA.108.183749

25. Deckert, V., Perségol, 1., Viens, 1., Lizard, G., Athias, A., Lallemant, C., et al. (1997) Inhibitors of arterial relaxation among components of human oxidized low-density lipoproteins cholesterol derivatives oxidized in position 7 are potent inhibitors of endothelium-dependent relaxation. Circulation 95, 723-731,

26. Wang, M., Long, W., Li, D., Wang, D., Zhong, Y., Mu, D., Song, J., Xia, M. (2017) Plasma 7-ketocholesterol levels and the risk of incident cardiovascular events. Heart 103, 1788-1794, http://dx.doi.org/10.1136/heartjnl-2016-310914. 
27. Song, J., Wang, D., Chen, H., Huang, X., Zhong, Y., Jiang, N., et al. (2017) Association of Plasma 7-Ketocholesterol With Cardiovascular Outcomes and Total Mortality in Patients With Coronary Artery Disease. Circ. Res. 120, 1622-1631, https://doi.org/10.1161/CIRCRESAHA.117.311049

28. Soler, M., Camacho, M., Escudero, J.R., Iñiguez, M.A., Vila, L. (2000) Human vascular smooth muscle cells but not endothelial cells express prostaglandin E synthase. Circ. Res. 87, 504-507.

29. Galán, M., Varona, S., Orriols, M., Rodríguez, J.A., Aguiló, S., Dilmé, J., et al. (2016) Induction of histone deacetylases (HDACs) in human abdominal aortic aneurysm: therapeutic potential of HDAC inhibitors. Dis. Model. Mech. 9, 541-552, https://doi.org/10.1242/dmm.024513

30. Helmschrodt, C., Becker, S., Schröter, J., Hecht, M., Aust, G., Thiery, J., Ceglarek, U. (2013) Fast LC-MS/MS analysis of free oxysterols derived from reactive oxygen species in human plasma and carotid plaque. Clin. Chim. Acta. 425, 3-8, https://doi.org/10.1016/j.cca.2013.06.022

31. Mukhopadhyay, S., Fellows, K., Browne, R.W., Khare, P., Krishnan Radhakrishnan, S., Hagemeier, J., et al. (2017) Interdependence of oxysterols with cholesterol profiles in multiple sclerosis. Mult. Scler. 23, 792-801, https://doi.org/10.1177/1352458516666187

32. National Cholesterol Education Program (NCEP) Expert Panel on Detection, Evaluation, and Treatment of High Blood Cholesterol in Adults (Adult Treatment Panel III). (2002) Third Report of the National Cholesterol Education Program (NCEP) Expert Panel on Detection, Evaluation, and Treatment of High Blood 
Cholesterol in Adults (Adult Treatment Panel III) final report. Circulation 106, 3143-3421.

33. Sudo R, Sato F, Azechi T, Wachi H. (2015) 7-Ketocholesterol-induced lysosomal dysfunction exacerbates vascular smooth muscle cell calcification via oxidative stress. Genes Cells. 20, 982-991. doi: 10.1111/gtc.12301.

34. Zarrouk A, Nury T, Karym EM, Vejux A, Sghaier R, Gondcaille C, et al. (2017) Attenuation of 7-ketocholesterol-induced overproduction of reactive oxygen species, apoptosis, and autophagy by dimethyl fumarate on $158 \mathrm{~N}$ murine oligodendrocytes. J Steroid Biochem Mol Biol. 169, 29-38. doi: 10.1016/j.jsbmb.2016.02.024

35. Van der Meij, E., Koning, G.G., Vriens, P.W., Peeters, M.F., Meijer, C.A., Kortekaas, K.E., et al. (2013) A Clinical Evaluation of Statin Pleiotropy: Statins Selectively and Dose-Dependently Reduce Vascular Inflammation. PLoS ONE 8, e53882, https://doi.org/10.1371/journal.pone.0053882

36. Minamino, T., Komuro, I., Kitakaze, M. (2010) Endoplasmic reticulum stress as a therapeutic target in cardiovascular disease. Circ. Res. 107, 1071-1082, https://doi.org/10.1161/CIRCRESAHA.110.227819

37. Binder P, Wang S, Radu M, Zin M, Collins L, Khan S, et al. (2019) Pak2 as a Novel Therapeutic Target for Cardioprotective Endoplasmic Reticulum Stress Response. Circ Res. 124, 696-711. doi: 10.1161/CIRCRESAHA.118.312829.

38. Prola A, Nichtova Z, Pires Da Silva J, Piquereau J, Monceaux K, Guilbert A, et al. (2019) Endoplasmic reticulum stress induces cardiac dysfunction through 
architectural modifications and alteration of mitochondrial function in cardiomyocytes. Cardiovasc. Res. 115, 328-342. doi: 10.1093/cvr/cvy197.

39. Oh-hashi, K., Koga, H., Ikeda, S., Shimada, K., Hirata, Y., Kiuchi, K. (2009) CRELD2 is a novel endoplasmic reticulum stress-inducible gene. Biochem. Biophys. Res. Commun. 387, 504-510, https://doi.org/10.1016/j.bbrc.2009.07.047

40. Christianson, J.C., Shaler, T.A., Tyler, R.E., Kopito, R.R. (2008) OS-9 and GRP94 deliver mutant alpha1-antitrypsin to the Hrd1-SEL1L ubiquitin ligase complex for ERAD. Nat. Cell. Biol. 10, 272-282, https://doi.org/10.1038/ncb1689

41. Horimoto, S., Ninagawa, S., Okada, T., Koba, H., Sugimoto, T., Kamiya, Y., et al. (2013) The unfolded protein response transducer ATF6 represents a novel transmembrane-type endoplasmic reticulum-associated degradation substrate requiring both mannose trimming and SEL1L protein. J. Biol. Chem. 288, 31517-31527, https://doi.org/10.1074/jbc.M113.476010

42. Qin, Y., Wang, Y., Liu, O., Jia, L., Fang, W., Du, J., Wei, Y. (2017) Tauroursodeoxycholic Acid Attenuates Angiotensin II Induced Abdominal Aortic Aneurysm Formation in Apolipoprotein E-deficient Mice by Inhibiting Endoplasmic Reticulum Stress. Eur. J. Vasc. Endovasc. Surg. 53, 337e345, https://doi.org/10.1016/j.ejvs.2016.10.026

43. Li, Y., Lu, G., Sun, D., Zuo, H., Wang, D.W., Yan, J. (2017) Inhibition of endoplasmic reticulum stress signaling pathway: A new mechanism of statins to suppress the development of abdominal aortic aneurysm. PLoS One. 12, e0174821. 10.1371/journal.pone.0174821. eCollection 2017. 
44. Ni, XQ., Lu, WW., Zhang, J.S., Zhu, Q., Ren, J.L., et al. (2018) Inhibition of endoplasmic reticulum stress by intermedin1-53 attenuates angiotensin II-induced abdominal aortic aneurysm in ApoE KO Mice. Endocrine. 62, 90-106. doi: $10.1007 / \mathrm{s} 12020-018-1657-6$.

45. Gosselet, F., Saint-Pol, J., Fenart, L. (2014) Effects of oxysterols on the blood-brain barrier: implications for Alzheimer's disease. Biochem. Biophys. Res. Commun. 446, 687-691, https://doi.org/10.1016/j.bbrc.2013.11.059

46. Marwarha, G., Ghribi, O. (2015) Does the oxysterol 27-hydroxycholesterol underlie Alzheimer's disease Parkinson's disease overlap?. Exp. Gerontol. 68, 13-18, https://doi.org/10.1016/j.exger.2014.09.013

47. Seet, R.C., Lee, C.Y., Loke, W.M., Huang, S.H., Huang, H., et al. (2011) Biomarkers of oxidative damage in cigarette smokers: which biomarkers might reflect acute versus chronic oxidative stress?. Free Radic Biol Med. 50, 1787-1793. doi: 10.1016/j.freeradbiomed.2011.03.019. Epub 2011 Mar 17.

48. Pedruzzi, E., Guichard, C., Ollivier, V., Driss, F., Fay, M., Prunet, C., Marie, J.C., et al. (2004) NAD(P)H oxidase Nox-4 mediates 7-ketocholesterol-induced endoplasmic reticulum stress and apoptosis in human aortic smooth muscle $\begin{array}{llll}\text { cells. Mol. } & \text { Cell. } & \text { Biol. 24, }\end{array}$ https://doi.org/10.1128/MCB.24.24.10703-10717.2004

49. Lutjohann, D., Breuer, O., Ahlborg, G., Nennesmo, I., Siden, A., Diczfalusy, U., et al. (1996) Cholesterol homeostasis in human brain: evidence for an age-dependent flux of 24S-hydroxycholesterol from the brain into the circulation. Proc. Natl. Acad. Sci. U S A. 93, 9799-9804. 
50. Glass, C.K., Saijo, K. (2008) Immunology: Oxysterols hold T cells in check. Nature 455, 40-41, https://doi.org/10.1038/455040a

51. Adiguzel Z, Arda N, Kacar O, Serhatli M, Gezer Tas S, Baykal AT, Baysal K, Acilan C. (2014) Evaluation of apoptotic molecular pathways for smooth muscle cells isolated from thoracic aortic aneurysms in response to oxidized sterols. Mol Biol Rep. 41, 7875-7584. doi: 10.1007/s11033-014-3681-9.

52. Sanda GM, Deleanu M, Toma L, Stancu CS, Simionescu M, Sima AV. (2017) Oxidized LDL-Exposed Human Macrophages Display Increased MMP-9 Expression and Secretion Mediated by Endoplasmic Reticulum Stress. J Cell Biochem. 118, 661-669. doi: 10.1002/jcb.25637.

53. Luchetti, F., Crinelli, R., Cesarini, E., Canonico, B., Guidi, L., et al. (2017) Endothelial cells, endoplasmic reticulum stress and oxysterols. Redox Biol. 13, 581-587. doi: 10.1016/j.redox.2017.07.014. Epub 2017 Jul 29.

54. Thomas, M., Gavrila, D., McCormick, M.L., Miller, F.J. Jr., Daugherty, A., Cassis, L.A. et al. (2006) Deletion of p47phox attenuates angiotensin II-induced abdominal aortic aneurysm formation in apolipoprotein E-deficient mice.

Circulation 114, 404-413, https://doi.org/10.1161/CIRCULATIONAHA.105.607168

55. Miller, F.J. Jr., Sharp, W.J., Fang, X., Oberley, L.W., Oberley, T.D., Weintraub, N.L. (2002) Oxidative stress in human abdominal aortic aneurysms: a potential mediator of aneurysmal remodeling. Arterioscler. Thromb. Vasc. Biol. 22, 560. 
56. Guzik, B., Sagan, A., Ludew, D., Mrowiecki, W., Chwała, M., Bujak-Gizycka, B., et al. (2013) Mechanisms of oxidative stress in human aortic aneurysms-association with clinical risk factors for atherosclerosis and disease severity. Int. J. Cardiol. 168, 2389-2396, https://doi.org/10.1016/j.ijcard.2013.01.278

57. Sinha, I., Sinha-Hikim, A.P., Hannawa, K.K., Henke, P.K., Eagleton,M.J., Stanley, J.C., et al. (2005) Mitochondrial-dependent apoptosis in experimental rodent abdominal aortic aneurysms. Surgery 138, 806-811, https://doi.org/10.1016/j.surg.2005.07.011

58. Bonnard C, Durand A, Peyrol S, Chanseaume E, Chauvin MA, Morio B, Vidal H, Rieusset J. (2008) Mitochondrial dysfunction results from oxidative stress in the skeletal muscle of diet-induced insulin-resistant mice. J Clin Invest. 118, 789-800. doi: 10.1172/JCI32601.

59. Chevtzoff C, Yoboue ED, Galinier A, Casteilla L, Daignan-Fornier B, Rigoulet M, Devin A. (2010) Reactive oxygen species-mediated regulation of mitochondrial biogenesis in the yeast Saccharomyces cerevisiae. J Biol Chem. 285, 1733-1742. doi: 10.1074/jbc.M109.019570.

60. Gabrielson, M., Vorkapic, E., Folkesson, M., Welander, M., Matussek, A., Dimberg, J., et al. (2016) Altered PPAR $\gamma$ Coactivator-1 Alpha Expression in Abdominal Aortic Aneurysm: Possible Effects on Mitochondrial Biogenesis. J. Vasc. Res. 53, 17-26, https://doi.org/10.1159/000446653 
61. Senft, D., Ronai, Z.A. (2015) UPR, autophagy, and mitochondria crosstalk underlies the ER stress response. Trends Biochem. Sci. 40, 141-148, https://doi.org/10.1016/j.tibs.2015.01.002

62. Razani, B., Feng, C., Coleman, T., Emanuel, R., Wen, H., Hwang, S., et al. (2012) Autophagy links inflammasomes to atherosclerotic progression. Cell Metab. 15, 534-544, https://doi.org/10.1016/j.cmet.2012.02.011

63. Lee, J., Giordano, S., Zhang, J. (2012) Autophagy, mitochondria and oxidative stress: cross-talk and redox signaling. Biochem. J. 441, 523-540, https://doi.org/10.1042/BJ20111451

64. Ni, X.Q., Lu, W.W., Zhang, J.S., Zhu, Q., Ren, J.L., et al. (2018) Inhibition of endoplasmic reticulum stress by intermedin1-53 attenuates angiotensin II-induced abdominal aortic aneurysm in ApoE KO Mice. Endocrine. 62, 90-106, doi: 10.1007/s12020-018-1657-6. 


\section{TABLES}

Table 1. Demographics and clinical data of individuals included in the study.

\begin{tabular}{|c|c|c|c|c|}
\hline & \multicolumn{2}{|c|}{ mRNA } & \multicolumn{2}{|c|}{ Plasma } \\
\hline & AAA & Normal Aorta & AAA & Blood donors \\
\hline $\mathbf{N}$ & 96 & 17 & 94 & 47 \\
\hline Age (years) & $70.9 \pm 6.5$ & $60.9 \pm 13.4$ & $71.0 \pm 6.7$ & $59.3 \pm 4.3$ \\
\hline Women $(\%, N)$ & $5.2(5)$ & $5.9(1)$ & $5.3(5)$ & $30(14)$ \\
\hline Aortic diameter (mm) & $64.1 \pm 1.4$ & - & $64.1 \pm 1.4$ & - \\
\hline Dyslipidemia $(\%, N)$ & $63.5(61)$ & $17.6(3)$ & $64.9(61)$ & - \\
\hline HTN $(\%, N)$ & $85.4(82)$ & $41.2(7)$ & $84.6(80)$ & 7.8 \\
\hline Diabetes $(\%, N)$ & $22.9(22)$ & $29.4(5)$ & $23.4(22)$ & - \\
\hline Smokers / ex-smokers $(\%, N)$ & $83(78)$ & $41.2(7)$ & $82(76)$ & 9.8 \\
\hline $\begin{array}{l}\text { Systolic blood pressure } \\
(\mathrm{mm} \mathrm{Hg}, \mathrm{N}=67)\end{array}$ & $139.8 \pm 19.3$ & - & $139.8 \pm 19.3$ & - \\
\hline Heart rate $(N=65)$ & $73 \pm 11.6$ & - & $73 \pm 11.6$ & - \\
\hline Glucose (mM, N=79) & $5.9 \pm 1.3$ & - & $5.5 \pm 0.9$ & - \\
\hline $\mathrm{TC}(\mathrm{mM})$ & - & - & $3.9 \pm 1.2$ & $5.6 \pm 0.8$ \\
\hline HDL-C (mM) & - & - & $1 \pm 0.3$ & $1.5 \pm 0.3$ \\
\hline LDL-C (mM) & - & - & $2.3 \pm 0.9$ & $3.5 \pm 0.7$ \\
\hline VLDL-C (mM) & - & - & $0.6 \pm 0.3$ & $0.6 \pm 0.3$ \\
\hline Triglicerides (mM) & - & - & $1.3 \pm 0.7$ & $1.4 \pm 0.7$ \\
\hline PAD & 47.9 & 0.0 & 48.4 & - \\
\hline BVD & 9.4 & 11.8 & 15.4 & - \\
\hline IHD & 24.0 & 0.0 & 27.5 & - \\
\hline COPD & 17.7 & 0.0 & 18.7 & - \\
\hline Antiplatelet users & 59.4 & 11.8 & 62.6 & - \\
\hline Statin users & 69.8 & 5.9 & 68.1 & 1.8 \\
\hline NSAID users & 4.2 & 0.0 & 2.2 & - \\
\hline Corticoid users & 7.3 & 0.0 & 4.4 & - \\
\hline Immuno-supressors users & 4.2 & 0.0 & 3.3 & - \\
\hline
\end{tabular}

Nominal variables are presented as \%. Continuous variables are presented as mean \pm SD. Due to the nature of normal aorta samples and plasma from donors some of the clinical characteristics are not always recorded or are unknown and infra-evaluation of them is probable. BVD, brain-vascular disease; COPD, chronic occlusive pulmonary disease; HTN, chronic hypertension; TC: total cholesterol; HDL-C: high-density lipoprotein-cholesterol; LDL-C: low density lipoprotein-cholesterol. PAD, peripheral artery disease; BVD, brain-vascular disease; IHD, ischemic heart disease; COPD, chronic occlusive pulmonary disease. 
Table 2. Associations between oxysterols and lipid profile variables in healthy donors and AAA patients.

\begin{tabular}{|c|c|c|c|c|c|c|}
\hline Variable & AAA vs HD & $\mathbf{n}$ & 7-KC & 24-S-HC & 27-HC & $7 \alpha-\mathrm{HC}$ \\
\hline $\begin{array}{c}\text { TC } \\
(\mathrm{mmol} / \mathrm{L})\end{array}$ & $\begin{array}{l}\text { AAA } \\
\text { HD }\end{array}$ & $\begin{array}{l}94 \\
47\end{array}$ & $\begin{array}{c}-0.0323 \\
(P<0.7573) \\
0.07300 \\
(P<0.6258)\end{array}$ & $\begin{array}{c}0.5762 \\
(P<0.0001) \\
0.5470 \\
(P<0.0001)\end{array}$ & $\begin{array}{c}\mathbf{0 . 4 6 2 7} \\
(\mathbf{P}<0.0001) \\
0.03066 \\
(\mathrm{P}<0.8379)\end{array}$ & $\begin{array}{c}0.1711 \\
(P<0.1010) \\
-0.0715 \\
(P<0.6326)\end{array}$ \\
\hline $\begin{array}{l}\text { HDL-C } \\
(\mathrm{mmol} / \mathrm{L})\end{array}$ & $\begin{array}{l}\text { AAA } \\
\text { HD }\end{array}$ & $\begin{array}{l}94 \\
47\end{array}$ & $\begin{array}{c}-0.0023 \\
(P<0.9825) \\
0.07150 \\
(P<0.6330)\end{array}$ & $\begin{array}{c}\mathbf{0 . 2 9 7 6} \\
(\mathrm{P}<\mathbf{0 . 0 0 3 6}) \\
0.1355 \\
(\mathrm{P}<0.3637)\end{array}$ & $\begin{array}{c}0.1385 \\
(P<0.1832) \\
0.2013 \\
(P<0.1749)\end{array}$ & $\begin{array}{c}\mathbf{0 . 2 2 9 4} \\
(\mathbf{P}<\mathbf{0 . 0 2 7}) \\
-0.0193 \\
(P<0.8974)\end{array}$ \\
\hline $\begin{array}{l}\text { LDL-C } \\
(\mathrm{mmol} / \mathrm{L})\end{array}$ & $\begin{array}{l}\text { AAA } \\
\text { HD }\end{array}$ & $\begin{array}{l}94 \\
47\end{array}$ & $\begin{array}{c}-0.0145 \\
(P<0.8896) \\
0.07491 \\
(P<0.6168)\end{array}$ & $\begin{array}{c}0.5730 \\
(P<0.0001) \\
0.5590 \\
(P<0.0001)\end{array}$ & $\begin{array}{c}\mathbf{0 . 4 8 3 9} \\
(\mathbf{P}<0.0001) \\
-0.04272 \\
(P<0.7756)\end{array}$ & $\begin{array}{c}0.1003 \\
(P<0.3388) \\
-0.0663 \\
(P<0.6577)\end{array}$ \\
\hline $\begin{array}{c}\text { TG } \\
(\mathrm{mmol} / \mathrm{L})\end{array}$ & $\begin{array}{l}\text { AAA } \\
\text { HD }\end{array}$ & $\begin{array}{l}94 \\
47\end{array}$ & $\begin{array}{c}-0.0842 \\
(P<0.4197) \\
-0.03966 \\
(P<0.7913)\end{array}$ & $\begin{array}{c}0.2828 \\
(P<0.0057) \\
0.1003 \\
(P<0.5024)\end{array}$ & $\begin{array}{c}0.2699 \\
(P<0.0085) \\
-0.008408 \\
(P<0.9553)\end{array}$ & $\begin{array}{c}0.1322 \\
(P<0.2066) \\
-0.0260 \\
(P<8621)\end{array}$ \\
\hline $\begin{array}{l}\text { VLDL-C } \\
(\mathrm{mmol} / \mathrm{L})\end{array}$ & $\begin{array}{l}\text { AAA } \\
\text { HD }\end{array}$ & $\begin{array}{l}94 \\
47\end{array}$ & $\begin{array}{c}-0.0819 \\
(P<0.4326) \\
-0.03609 \\
(P<0.8097)\end{array}$ & $\begin{array}{c}\mathbf{0 . 2 8 5 6} \\
(\mathbf{P}<\mathbf{0 . 0 0 5 3}) \\
0.1011 \\
(P<0.4988)\end{array}$ & $\begin{array}{c}\mathbf{0 . 2 6 9 8} \\
(\mathbf{P}<0.0085) \\
-0.01043 \\
(\mathrm{P}<0.9445)\end{array}$ & $\begin{array}{c}0.1328 \\
(P<0.2046) \\
-0.0266 \\
(P<8591)\end{array}$ \\
\hline $\begin{array}{c}\text { AA } \\
\text { diameter }\end{array}$ & AAA & 94 & $\begin{array}{c}-0.191 \\
(P<0.15)\end{array}$ & $\begin{array}{c}-0.119 \\
(P<0.246)\end{array}$ & $\begin{array}{c}-0.196 \\
(P<0.107)\end{array}$ & $\begin{array}{c}-0.129 \\
(P<0.208)\end{array}$ \\
\hline
\end{tabular}

HD: healthy donor; AAA: aneurysm patients; $\mathrm{KC}$ : ketocholesterol; $\mathrm{HC}$ : hydroxycholesterol; TC: total cholesterol; HDL-C: high-density lipoprotein-cholesterol; LDL-C: low density lipoprotein-cholesterol. The Pearson product-moment correlation coefficient is shown (r). The significant $\mathrm{P}$ values are shown in bold. 


\section{Figure 1}

A

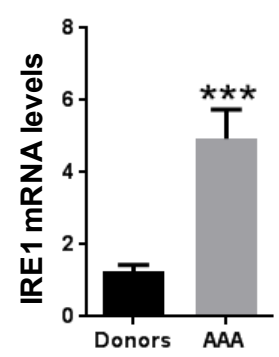

E

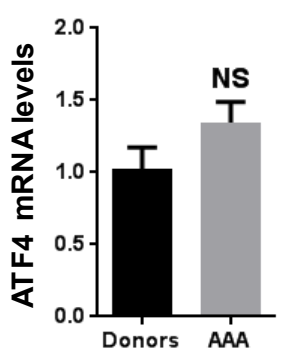

B

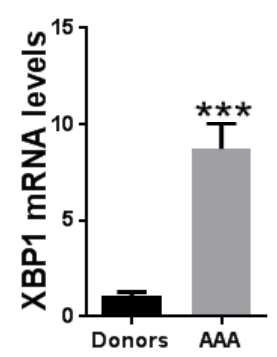

$\mathbf{F}$

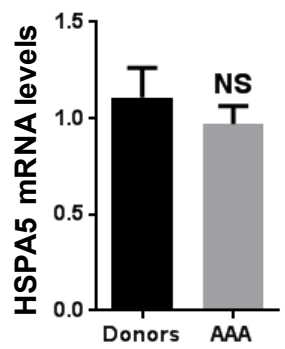

C

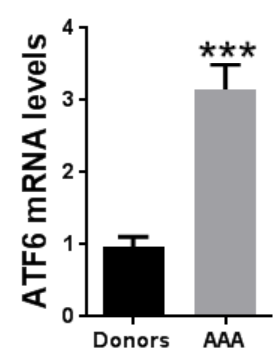

G

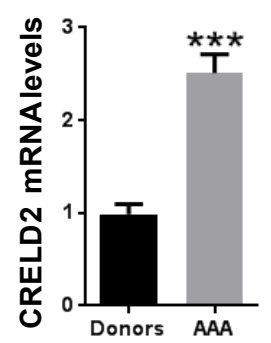

D

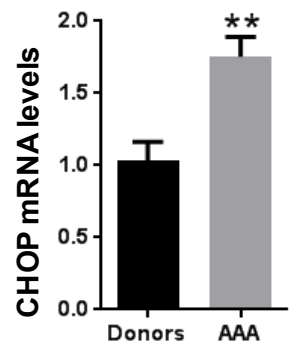

H

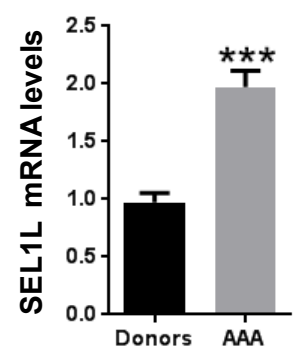

Figure 1. Endoplasmic reticulum stress markers expression is altered in human abdominal aortic aneurysm (AAA). A-H) Human abdominal aortic mRNA levels of IRE-1, XBP-1, ATF4, ATF6, HSPA5, CHOP, CRELD2 and SEL1L measured by quantitative real Time PCR analysis and normalized to $\beta$-actin in healthy donors $(\mathrm{n}=17)$ and patients (AAA) $(\mathrm{n}=96)$. Results are expressed as mean \pm SEM. $* * p<0.01 v s$. AAA; $* * * p<0.001 v s$. AAA. 


\section{Figure 2}

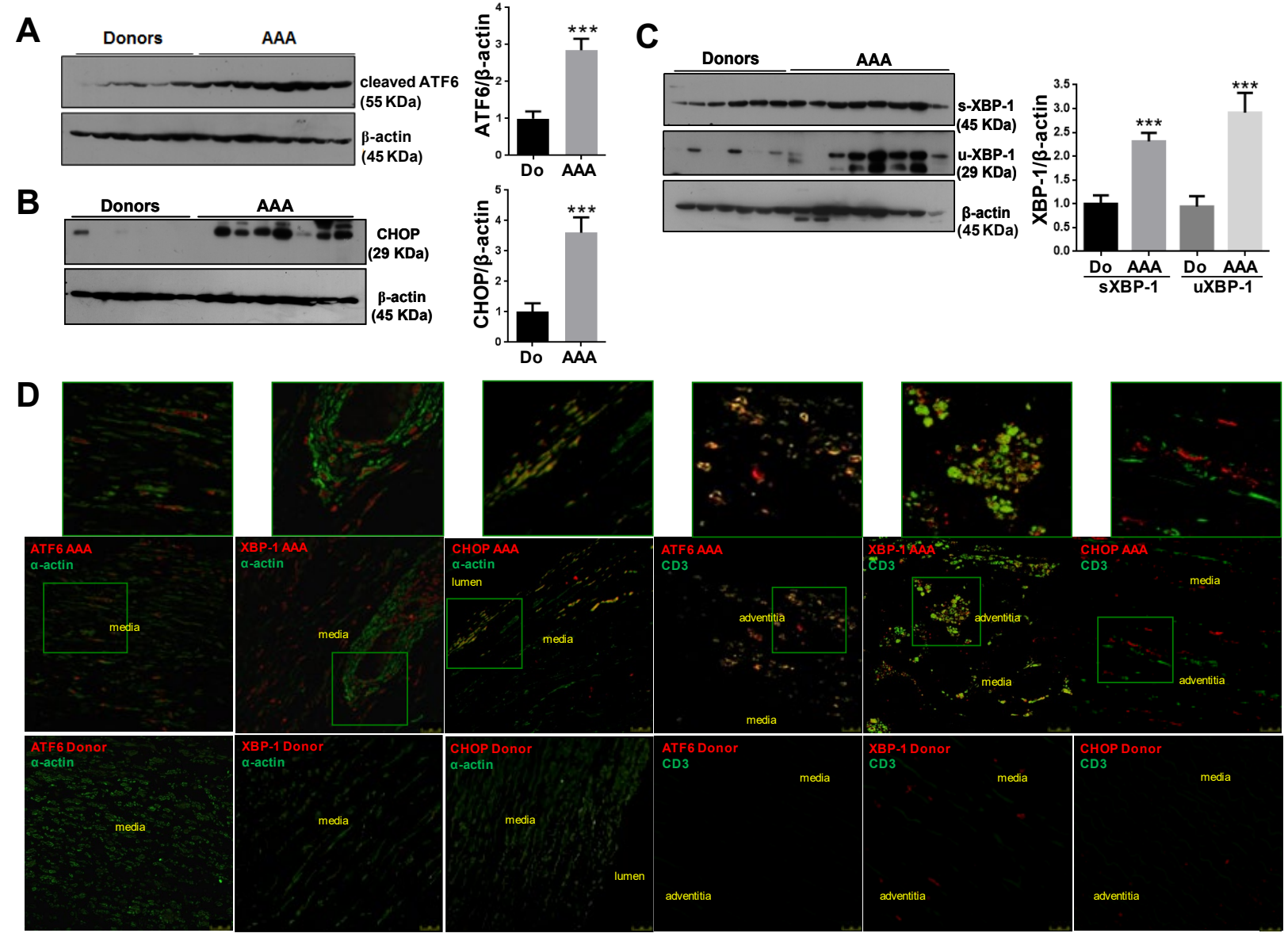

Figure 2. Endoplasmic reticulum stress markers expression in human abdominal aortic aneurysm (AAA) and co-localization with VSMC and lymphocytes T. A-C) Representative Western blot analysis of cleaved ATF6, CHOP and XBP-1 (unspliced and spliced isoforms) and their corresponding quantitative histogram below (AAA: $\mathrm{n}=15$ and Donors: $\mathrm{n}=10$ ). Results are expressed as mean \pm SEM. ${ }^{* *} p<0.01 v s$. AAA; $* * * p<0.001 v s$. AAA. D) Representative images of immunofluorescence assays performed in abdominal aorta sections targeting ATF6, XBP-1, or CHOP (red) and $\alpha$-actin or CD3 positive cells (green) as indicated in AAA patients (upper panels) and donors (lower panels) analyzed by confocal microscopy ( $\mathrm{N}=8-10$; Scale bars: $25 \mu \mathrm{m})$. 


\section{Figure 3}

A

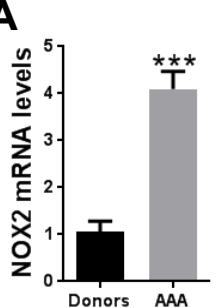

B
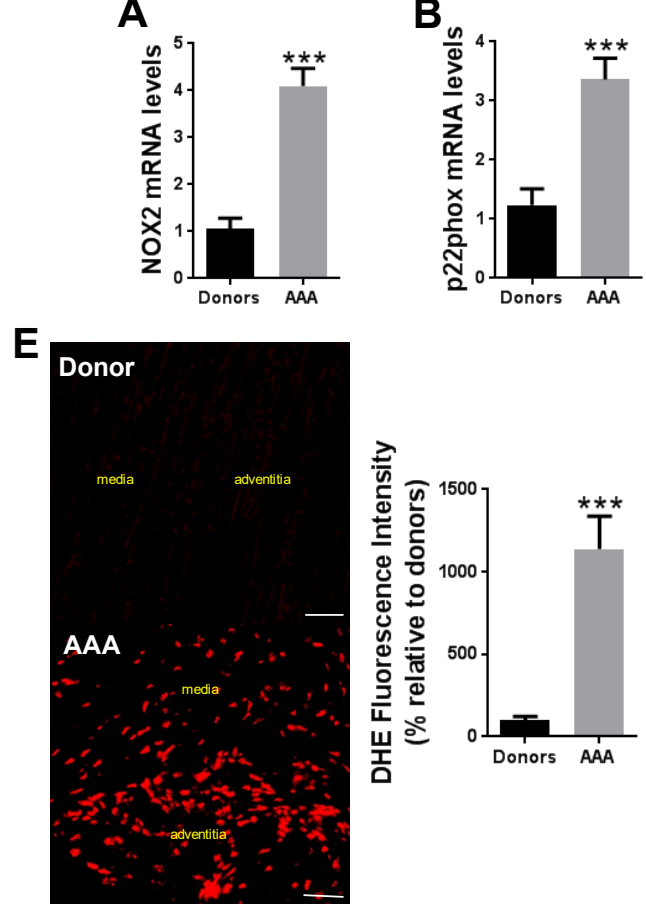

G
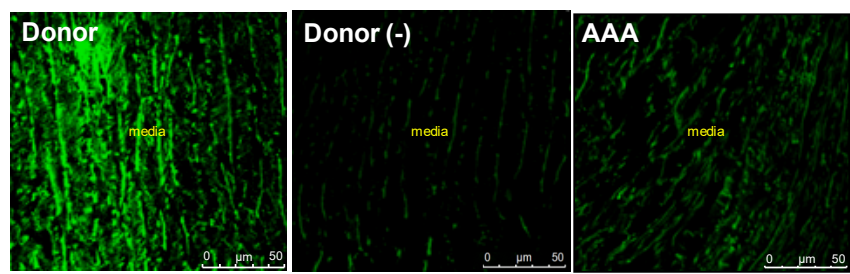

AAA (-)

C

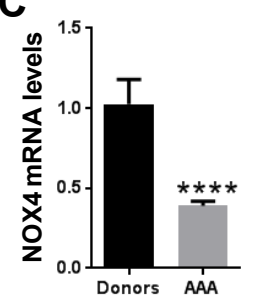

$\mathbf{F}$

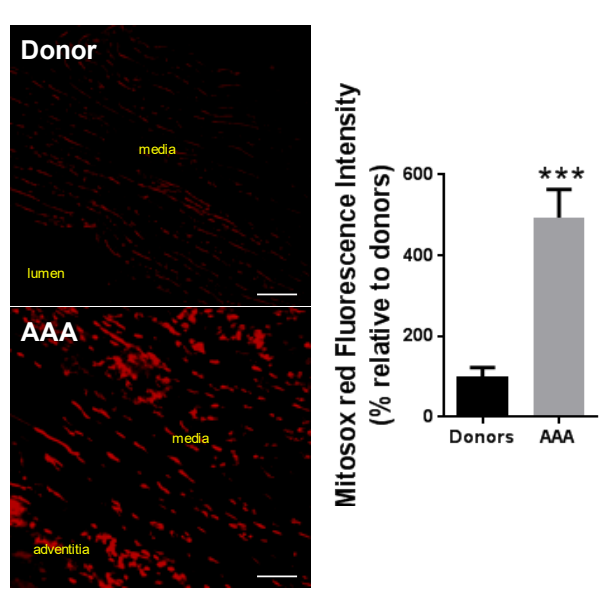

D

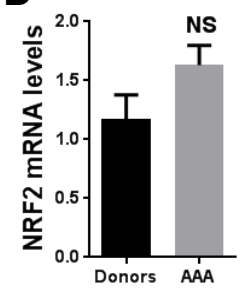

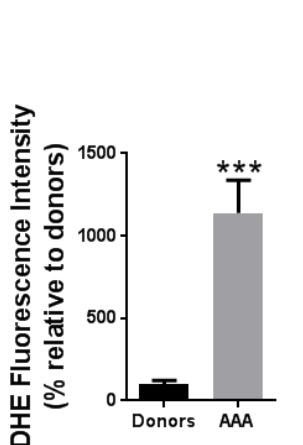

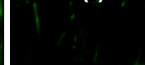

media

Figure 3. Oxidative stress is exacerbated in AAA. A-D) NOX2, p22phox, NOX4, and Nuclear factor erythroid 2-related factor 2 (NRF2) mRNA levels quantified by real Time PCR analysis and normalized to $\beta$-actin in abdominal aortas from donors $(n=17)$ and AAA patients $(n=96)$. E-F) Representative abdominal aortic sections from frozen tissue preserved in OCT stained with fluorescent probes dihydroethidium (DHE) (E) or mitosox red (F). The histograms show the quantification of the fluorescence intensity $(\mathrm{n}=10$; bar: $50 \mu \mathrm{m}) . \mathbf{G})$ Representative abdominal aortic sections stained with the fluorescent probe Mitotracker green labelling mitochondrial mass $(\mathrm{n}=10$; bar: $75 \mu \mathrm{m})$. Results are expressed as mean \pm SEM. ${ }^{* * *} p<0.001 v s$. AAA. 


\section{Figure 4}

A

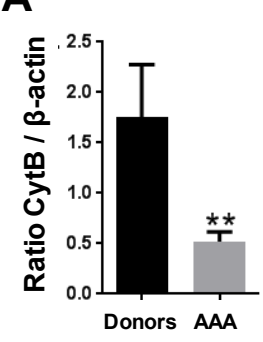

$\mathbf{F}$

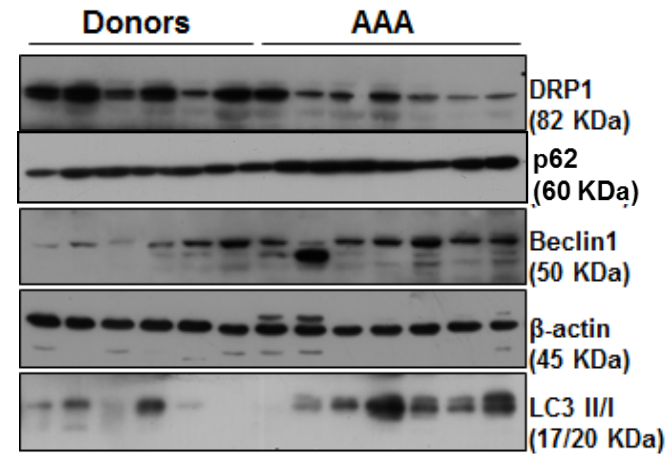

B

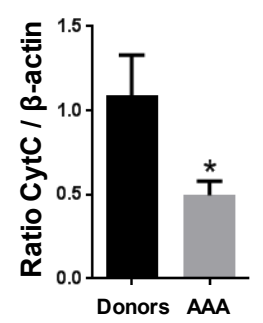

C

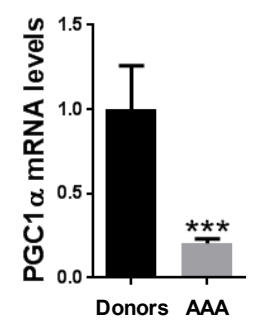

D

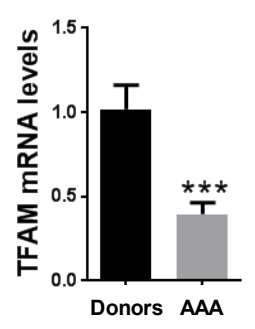

E

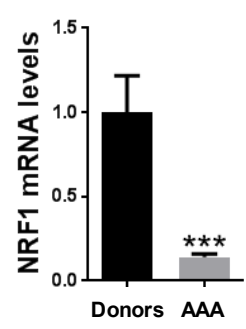

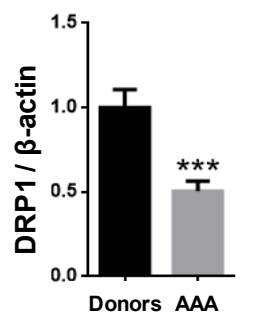
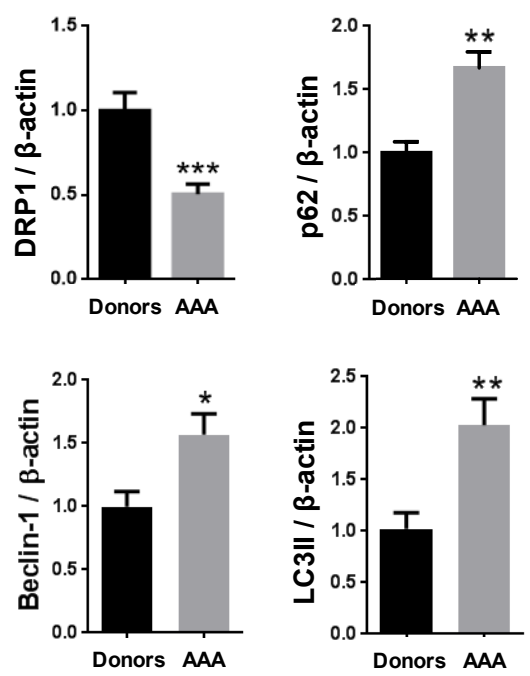

Figure 4. Mitochondrial biogenesis and mitophagy / autophagy alterations in AAA. A-B) Graphs showing the ratios between Cytochrome B or cytochrome C oxidase (MT-CO3 gene) and $\beta$-actin. C-E) PGC1 $\alpha$, TFAM, and NRF1 mRNA levels quantified by real Time PCR analysis and normalized to GAPDH in abdominal aortas from donors $(\mathrm{n}=17)$ and AAA patients $(\mathrm{n}=96)$. F) Representative Western blot analysis of DRP-1, p62, Beclin-1, and LC3II and their corresponding quantitative histogram (AAA: $n=15$ and Donors: $n=10$ ). Results are expressed as mean \pm SEM. ${ }^{* *} p<0.01 v s$. AAA; $* * * p<0.001 v s$. AAA. 


\section{Figure 5}

A

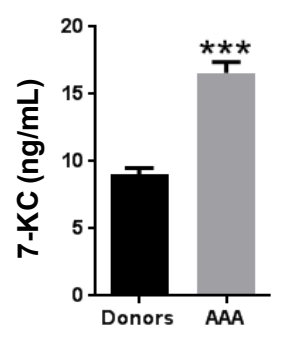

$\mathbf{F}$

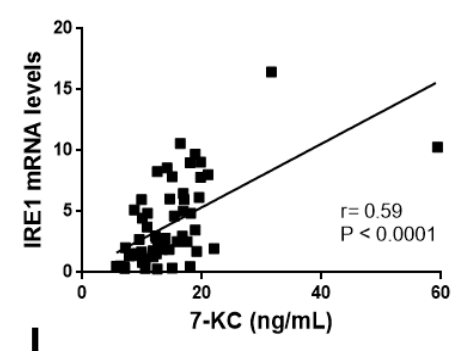

I

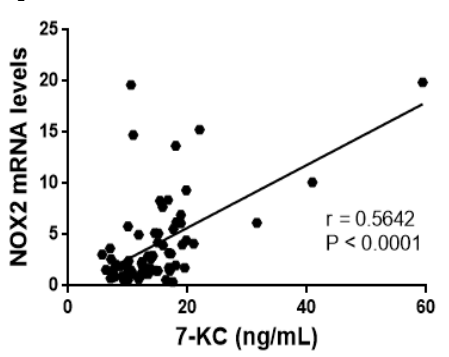

C

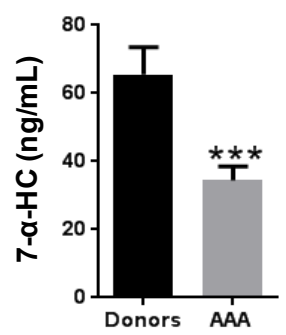

G
D

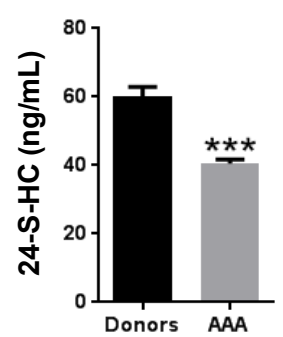

H
E

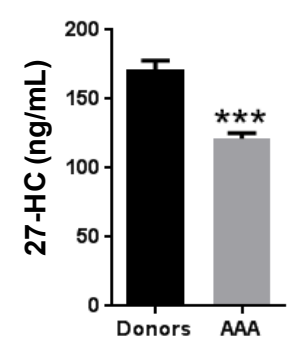

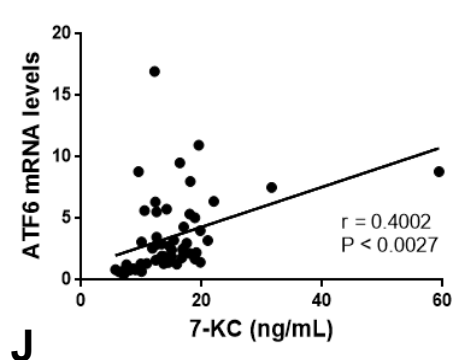
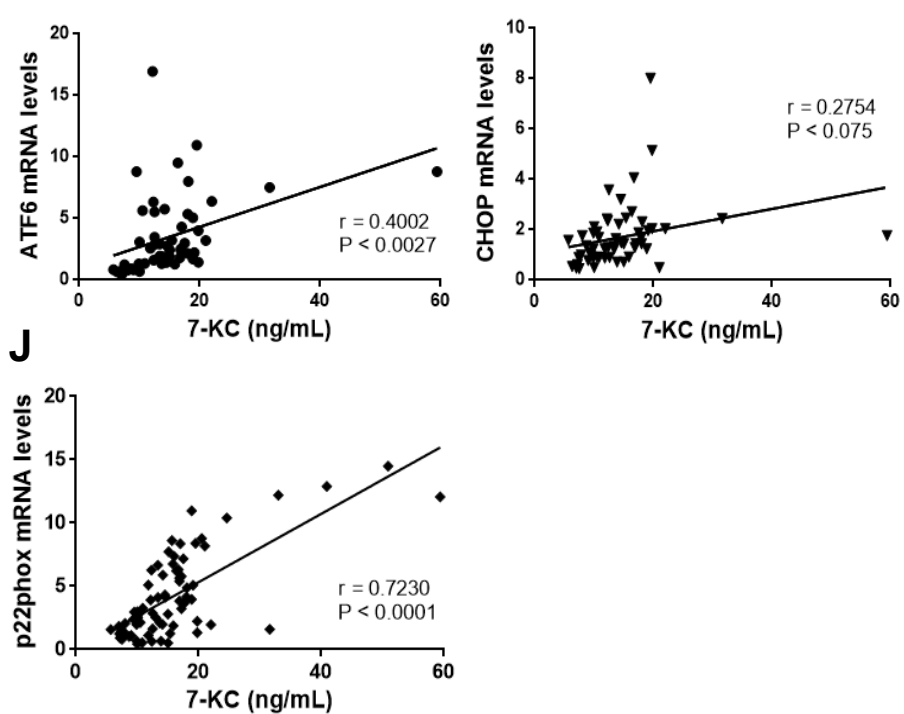

Figure 5. Oxysterols levels are altered in AAA patients. A-E) Histograms representing the values of $7-\mathrm{KC}, 7 \alpha-\mathrm{HC}, 7 \beta-\mathrm{HC}, 24-\mathrm{HC}$, and $27-\mathrm{HC}$ in nanogram per milliliter in plasma of healthy donors and AAA patients. Results are expressed as mean \pm SEM. The p-values from a Mann-Whitney test are provided on the donors versus AAA comparison: ${ }^{* * *} p<0.001 v$ s. AAA. F-H) Graphs showing the correlation analysis between IRE1, ATF6 and CHOP mRNA levels and 7-KC levels in AAA patients $(\mathrm{n}=75)$. I-J) Graphs showing the correlation analysis between NOX2 or p22phox mRNA levels and 7-KC levels in AAA patients $(\mathrm{n}=78)$. The $\mathrm{r}$ and $\mathrm{p}$-values are obtained by performing the Pearson correlation coefficient test. 


\section{Figure 6}
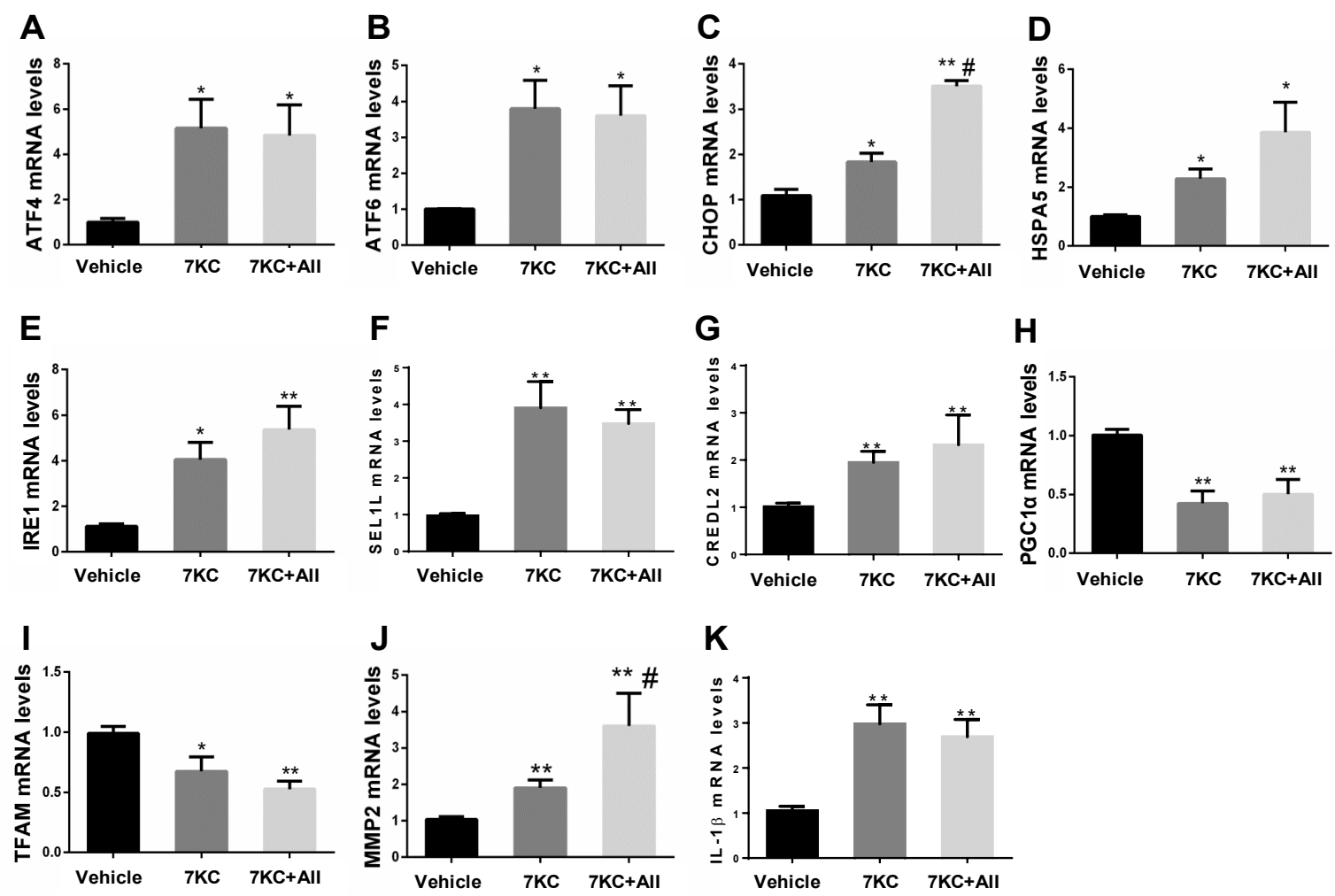

K

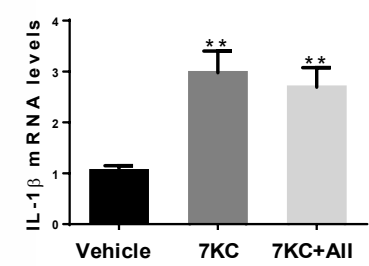

Figure 6. The exposure to 7-KC triggers ER stress response in human VSMC and the addition of angiotensin II has a synergistic effect. A-G) mRNA levels of HSPA5, ATF4, ATF6, IRE1, CHOP, SEL1L and CREDL2; H-K) mRNA levels of PGC1 alpha, TFAM, and NRF1 normalized to GAPDH in human VSMC stimulated with $7-\mathrm{KC}\left(15 \times 10^{-6} \mathrm{M}\right)$ for $24 \mathrm{~h}$ in the presence or absence of angiotensin II (AII, $\left.10^{-7} \mathrm{M}\right)$ for $48 \mathrm{~h}$. Values are shown as mean $\pm \mathrm{SEM}(\mathrm{n}=6$; ${ }^{*} p<0.01$ vs. vehicle; $\# p<0.05$ vs $\left.7-\mathrm{KC}\right)$. 


\section{Supplementary Figure S1}
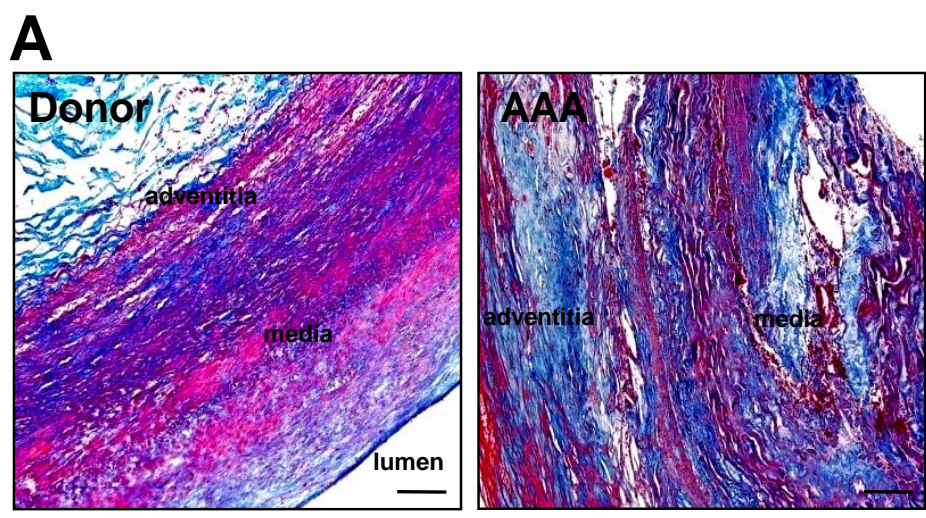

B
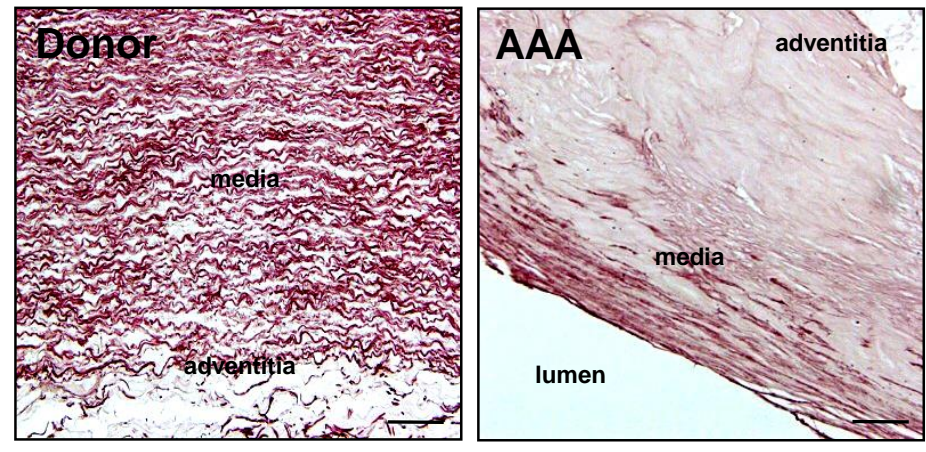

Figure S1. Extracellular matrix in human abdominal aortic aneurysm (AAA). A) Representative aortic sections stained with Masson's trichrome captured with 10x objectives (bars: $200 \mu \mathrm{m}$ ). B) Representative aortic sections stained with orcein showing the morphology of disorganized and disrupted elastic fibers in abdominal aortic wall from donors and in aneurysmatic wall of AAA patients (20x, bars: $50 \mu \mathrm{m})$. 


\section{Supplementary Figure 2}

A
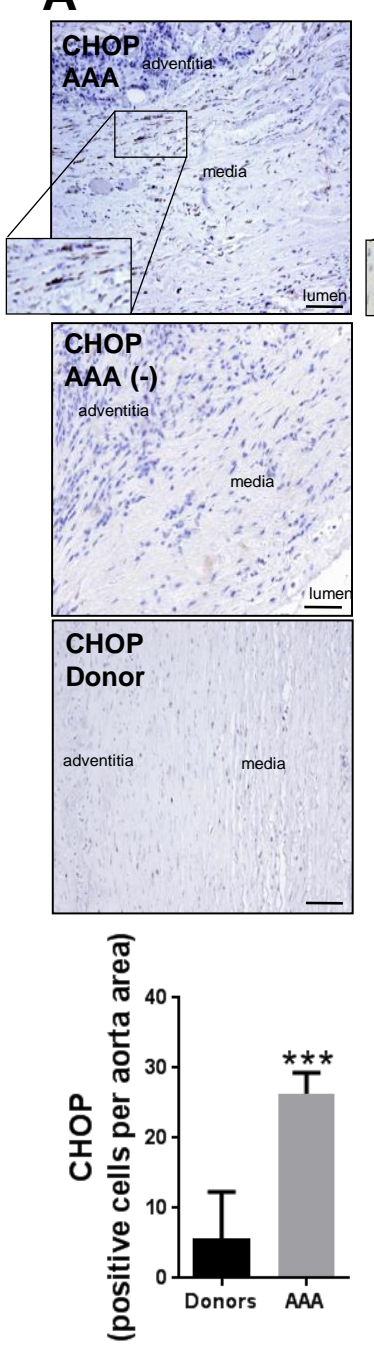

B
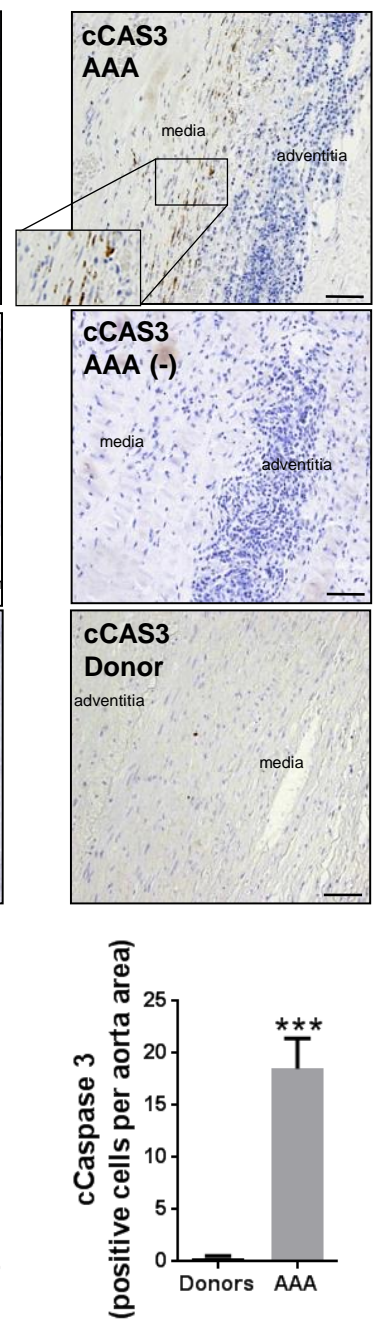

C
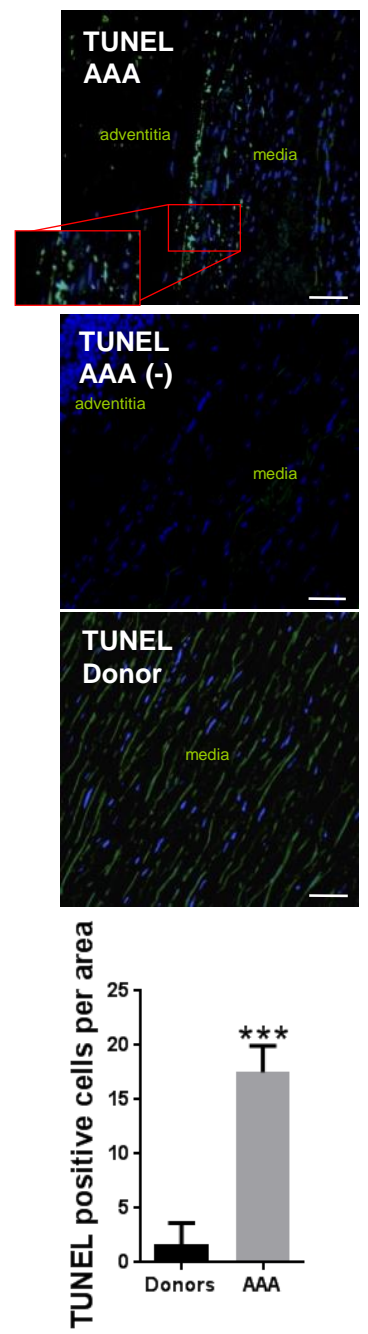

D
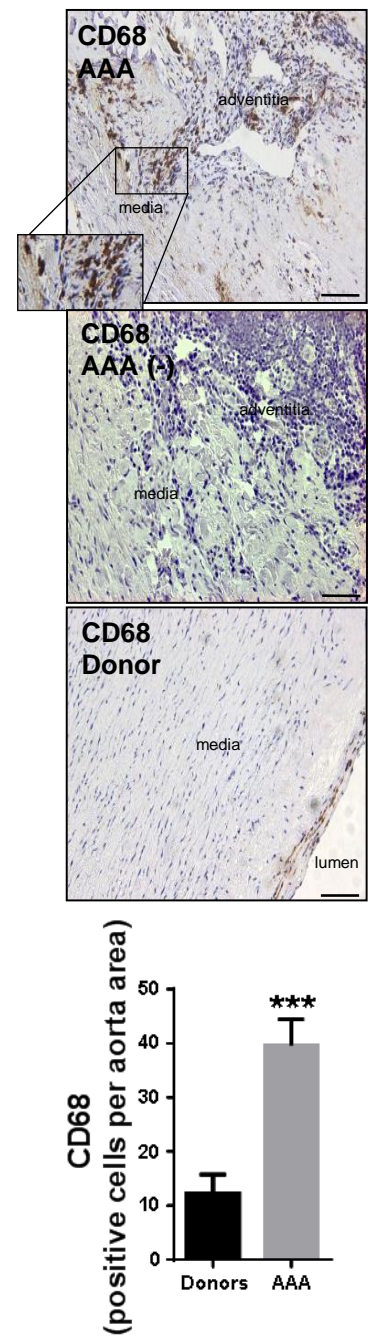

Figure S2. Apoptotic markers and macrophages infiltration in human abdominal aortic aneurysm (AAA). A-B) Representative images of immunohistochemistry assay and quantification against $\mathrm{CHOP}$ and cleaved caspase 3 (cCAS3) in haematoxylin counterstained aortic samples of AAA patients and donors $(\mathrm{n}=8-10$; Scale bar: $50 \mu \mathrm{m})$. C) Representative images of TUNEL assay and quantification performed in sections of paraffined abdominal aortas from AAA patients and donors ( $n=8-10$; Scale bar: $50 \mu \mathrm{m})$ analyzed by fluorescence microscopy. D) Representative images of immunohistochemistry assay against the macrophages marker CD68 in haematoxylin counterstained aortic samples of AAA patients and donors ( $n=8-10$; Scale bar: $50 \mu \mathrm{m})$. The histograms show the quantification of the number of positive cells per aortic area (20x). Results are expressed as mean \pm SEM. $* * p<0.01 v s$. AAA. 
Supplementary figure 3 .

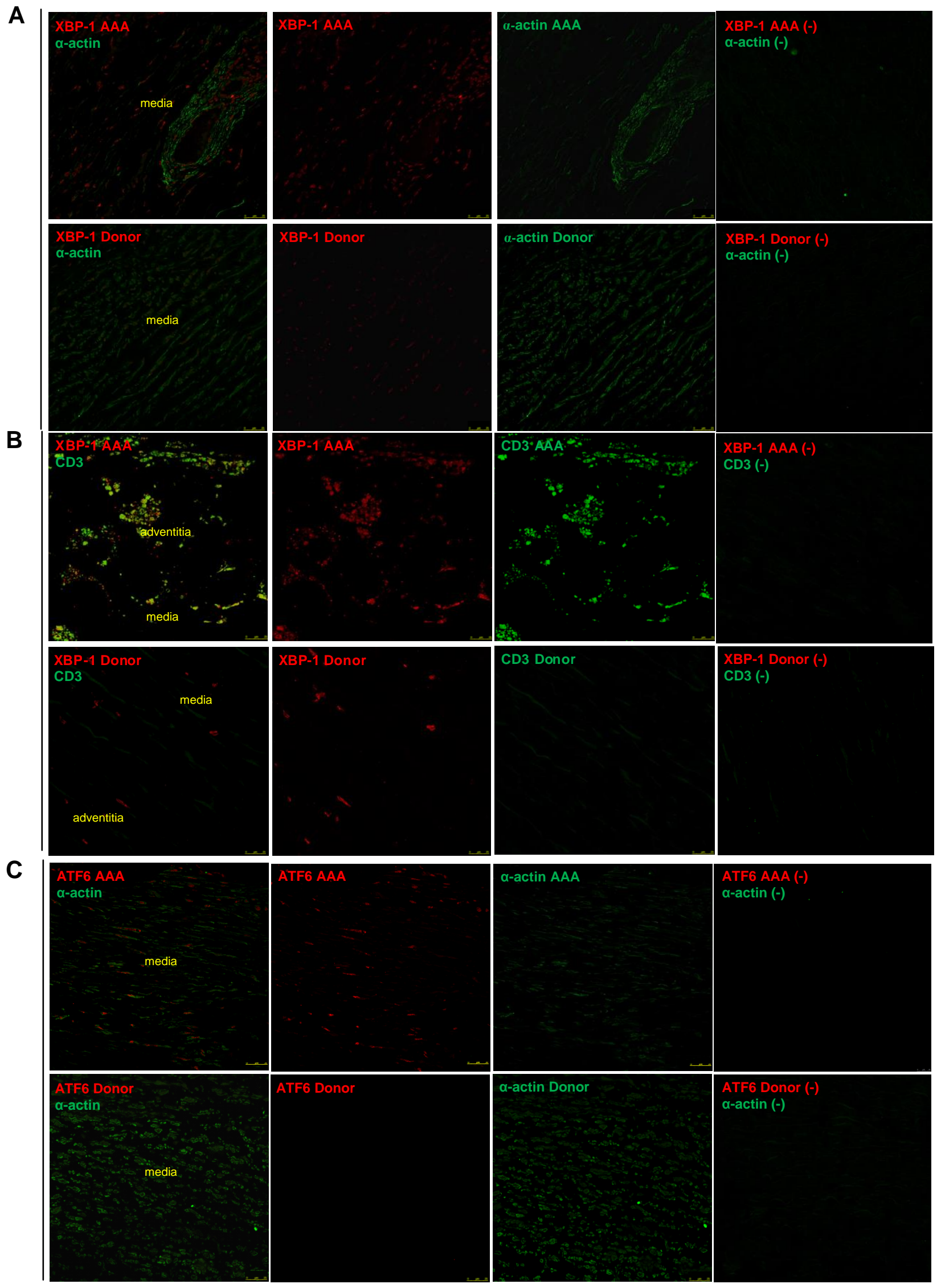



Figure S3. A,C,E) Representative images showing merge, single staining of XBP-1, ATF6 or CHOP (red) and $\alpha$-actin (green) and their respective negative control in AAA patients (upper panels) and donors (lower panels). B,D,F) Representative images showing merge, single staining of XBP-1, ATF6 or CHOP (red) and CD3 (green) and their negative control in AAA patients (upper panels) and donors (lower panels) analyzed by confocal microscopy ( $\mathrm{N}=8-10$; Scale bars: $25 \mu \mathrm{m})$. 


\section{Supplementary Figure 4}

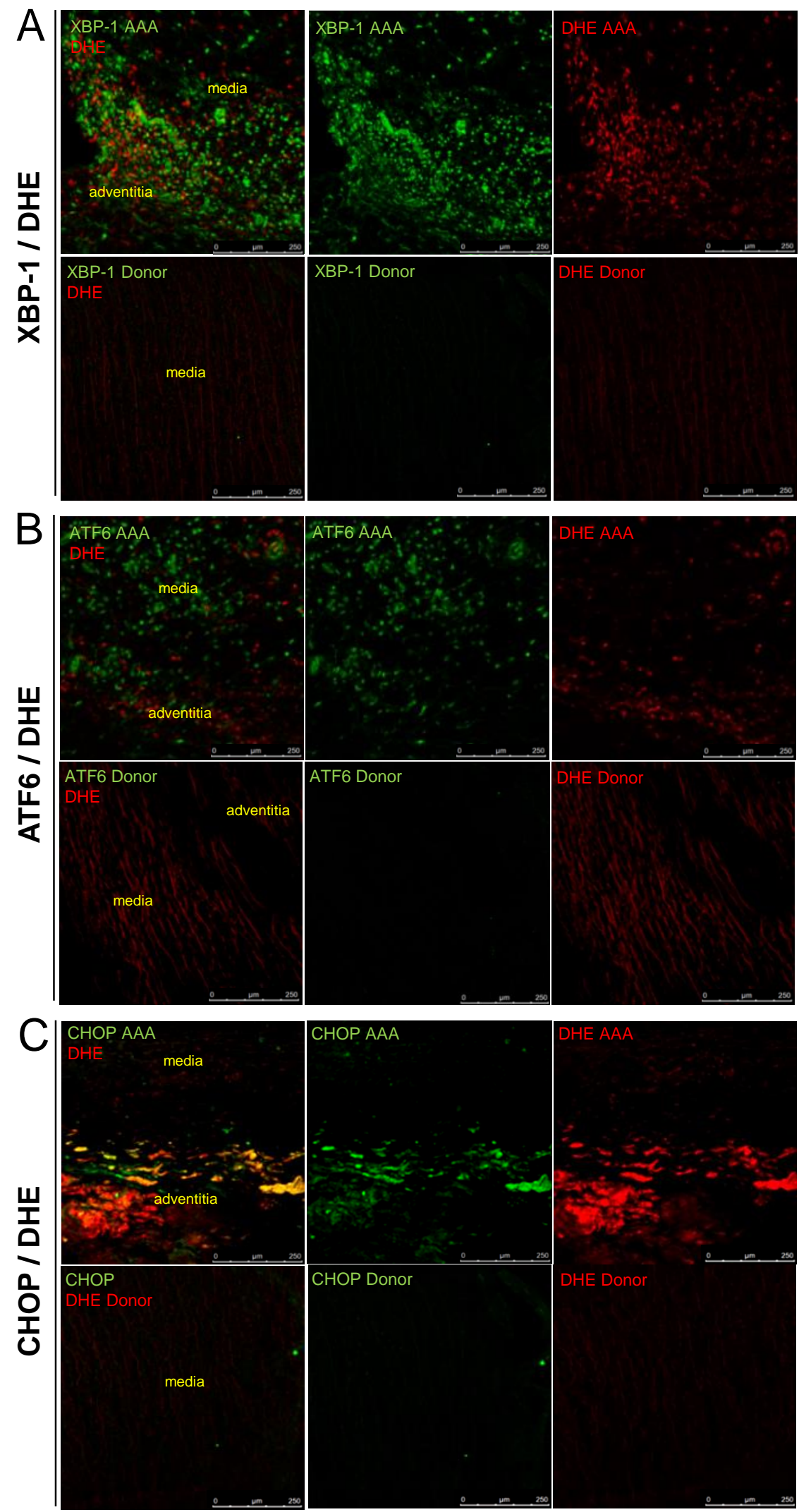

Figure S4. Colocalization of ER stress markers expression and superoxide anion. A-C) Representative images of immunofluorescence assay against XBP-1, ATF6 and CHOP (green) and DHE staining (red) in frozen aortic sections of AAA patients and donors ( $\mathrm{n}=8-10$; Scale bar: $250 \mu \mathrm{m})$. 


\section{Supplementary Figure 5}

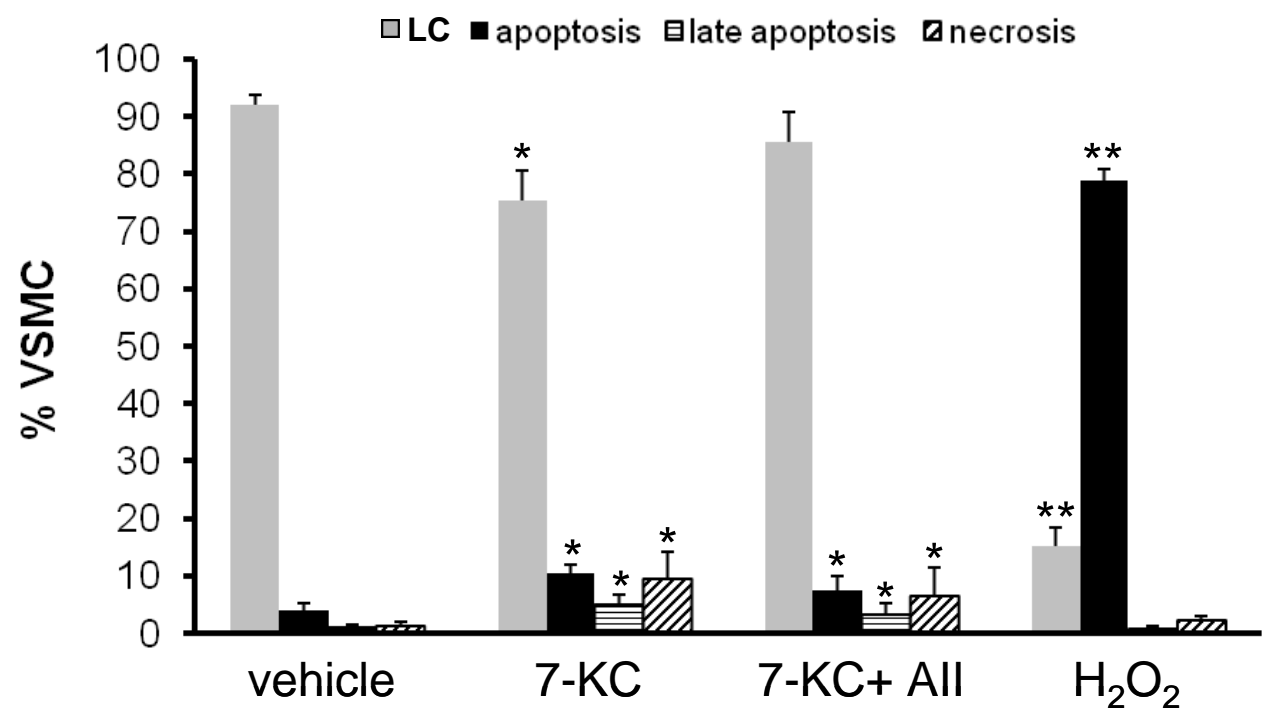

Figure S5. Evaluation of apoptosis by Annexin V and 7-AAD staining. VSMC were exposed to $7-\mathrm{KC}\left(15 \times 10^{-6} \mathrm{M}\right)$ for $24 \mathrm{~h}$ in the presence or absence of AII $\left(10^{-7} \mathrm{M}\right)$. Percentage of live cells (LC), early apoptotic (apoptosis), late apoptotic, and necrotic cells are represented. Values are shown as mean \pm SEM $(n=4 ; * p<0.01$ vs. vehicle; \# $p<$ 0.05 vs $7-\mathrm{KC})$. 


\section{Supplementary Figure 6}
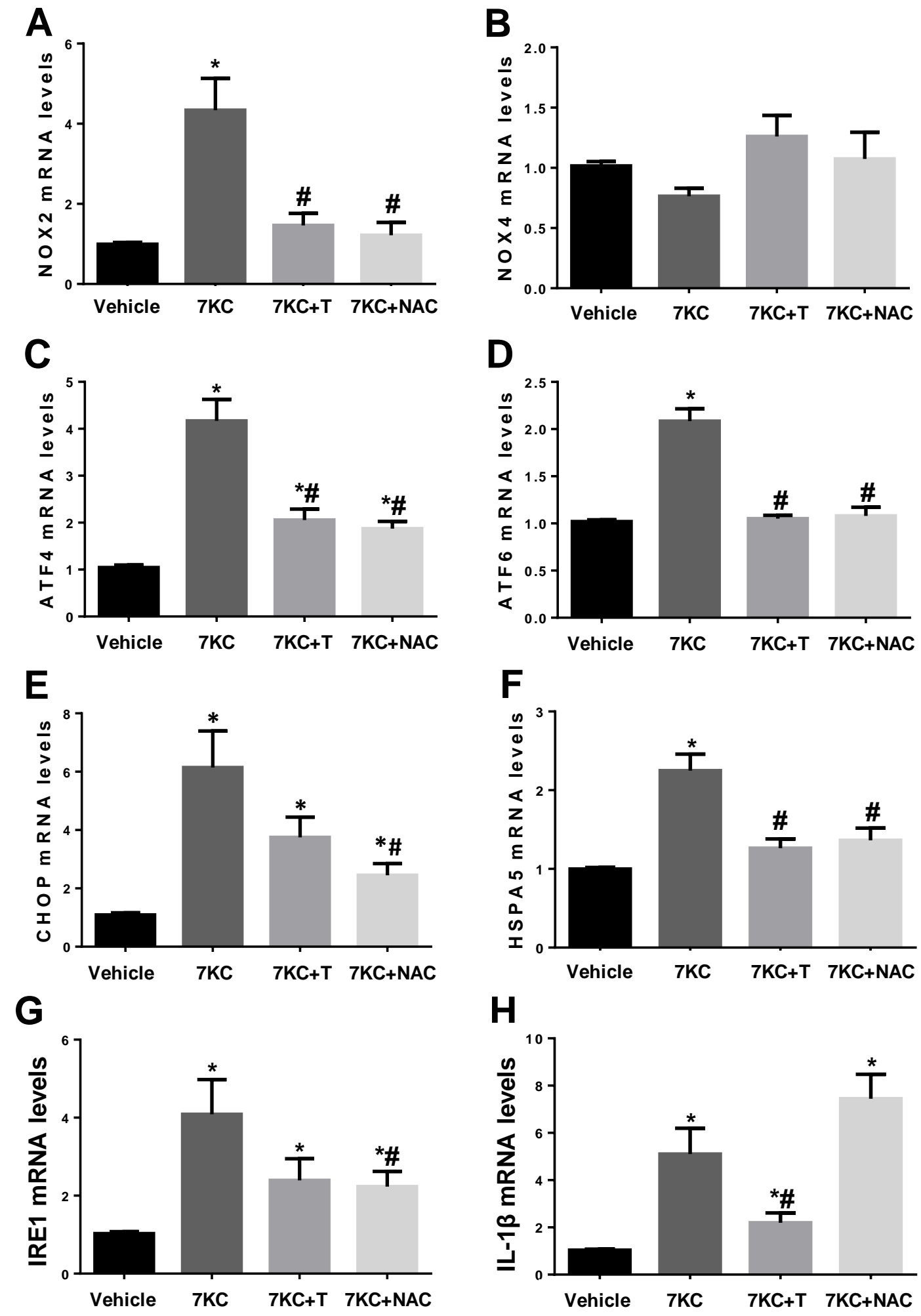

Figure S6. Protective role of antioxidants in response to incubation with 7-KC. A-E) mRNA levels of NOX2, NOX4, ATF4, ATF6, CHOP, HSPA5, IRE1 and IL-1 $\beta$, normalized to GAPDH, in human VSMC triggered with 7-KC $\left(15 \times 10^{-6} \mathrm{M}\right)$ for $24 \mathrm{~h}$ in the presence or absence of NAC $(10 \mathrm{mM})$ or Tempol $\left(\mathrm{T}, 10^{-6} \mathrm{M}\right)$ for $24 \mathrm{~h}$. Values are shown as mean $\pm \operatorname{SEM}\left(\mathrm{n}=6 ;{ }^{*} p<0.01\right.$ vs. vehicle; $\# p<0.05$ vs $\left.7-\mathrm{KC}\right)$. 
Table S1. Logistic regression analysis. OR: Odd Ratio; 95\% CI: 95 confidence interval. ${ }^{*} \mathrm{P} \leq 0.05$ vs. Donors: statistically significant.

\begin{tabular}{|c|c|c|}
\hline & OR $(95 \% \mathrm{Cl})$ & P-value \\
\hline ATF6 & $2.396(1.338-4.291)$ & $0.003^{*}$ \\
\hline \multicolumn{3}{|c|}{ Association after adjusting for: } \\
\hline Age & $3.528(1.232-10.101)$ & $0.019^{*}$ \\
\hline Sex & $4.023(1.429-11.326)$ & $0.008^{*}$ \\
\hline Smoking & $3.45(1.204-9.880)$ & $0.021^{*}$ \\
\hline Hypertension & $3.788(1.354-10.592)$ & $0.011^{*}$ \\
\hline Type 2 diabetes & $4.217(1.489-11.937)$ & $0.007^{*}$ \\
\hline All factors & $2.858(1.000-8.169)$ & $0.05^{\star}$ \\
\hline ERN1 & $2.341(1.256-4.363)$ & $0.007^{\star}$ \\
\hline \multicolumn{3}{|c|}{ Association after adjusting for: } \\
\hline Age & $2.54(1.228-5.255)$ & $0.012^{*}$ \\
\hline Sex & $2.326(1.246-4.342)$ & $0.008^{*}$ \\
\hline Smoking & $2.400(1.192-4.835)$ & $0.014^{*}$ \\
\hline Hypertension & $2.868(1.355-6.07)$ & $0.006^{*}$ \\
\hline Type 2 diabetes & $2.635(1.309-5.303)$ & $0.007^{\star}$ \\
\hline All factors & 3.378 (1.165-9.798) & $0.025^{*}$ \\
\hline CHOP & $7.408(1.702-32.873)$ & $0.008^{*}$ \\
\hline \multicolumn{3}{|c|}{ Association after adjusting for: } \\
\hline Age & $4.705(1.035-21.387)$ & $0.045^{\star}$ \\
\hline Sex & $7.743(1.725-34.762)$ & $0.008^{*}$ \\
\hline Smoking & $5.204(1.241-21.829)$ & $0.024^{\star}$ \\
\hline Hypertension & $5.554(1.319-23.385)$ & $0.019^{*}$ \\
\hline Type 2 diabetes & $7.942(1.708-36.934)$ & $0.008^{*}$ \\
\hline All factors & $3.800(0.624-23.131)$ & 0.147 \\
\hline XBP-1 & $3.692(1.651-8.256)$ & $0.001^{*}$ \\
\hline \multicolumn{3}{|c|}{ Association after adjusting for: } \\
\hline Age & $3.150(1.496-6.635)$ & $0.003^{*}$ \\
\hline Sex & $3.755(1.652-8.535)$ & $0.002^{*}$ \\
\hline Smoking & $2.910(1.367-6.199)$ & $0.006^{*}$ \\
\hline Hypertension & $3.031(1.496-6.635)$ & $0.005^{\star}$ \\
\hline Type 2 diabetes & $3189(1.494-6.806)$ & $0.003^{*}$ \\
\hline All factors & $2.481(1.183-5.203)$ & $0.016^{*}$ \\
\hline NOX2 & $1.958(1.134-3.382)$ & $0.016^{*}$ \\
\hline \multicolumn{2}{|c|}{ Association after adjusting for: } & $0.010^{*}$ \\
\hline Sex & $2.010(1.185-3.409)$ & $0.010^{*}$ \\
\hline Smoking & $1.691(1.027-2.785)$ & $0.039^{*}$ \\
\hline Hypertension & $1.757(1.042-2.963)$ & $0.035^{\star}$ \\
\hline Type 2 diabetes & $1.904(1.128-3.214)$ & $0.016^{*}$ \\
\hline All factors & $2.543(1.237-5.228)$ & $0.011^{*}$ \\
\hline p22phox & $2.396(1.338-4.291)$ & $0.003^{*}$ \\
\hline \multicolumn{2}{|c|}{ Association after adjusting for: } & $0.006^{*}$ \\
\hline Sex & $2.397(1.340-4.291)$ & $0.003^{*}$ \\
\hline Smoking & $2.308(1.276-4.176)$ & $0.006^{*}$ \\
\hline Hypertension & 2.65 (1.339-5.247) & $0.005^{\star}$ \\
\hline Type 2 diabetes & $2.408(1.330-4.358)$ & $0.004^{*}$ \\
\hline All factors & $5.195(1.623-16.635)$ & $0.006^{*}$ \\
\hline
\end{tabular}


Table S2. Logistic regression analysis.

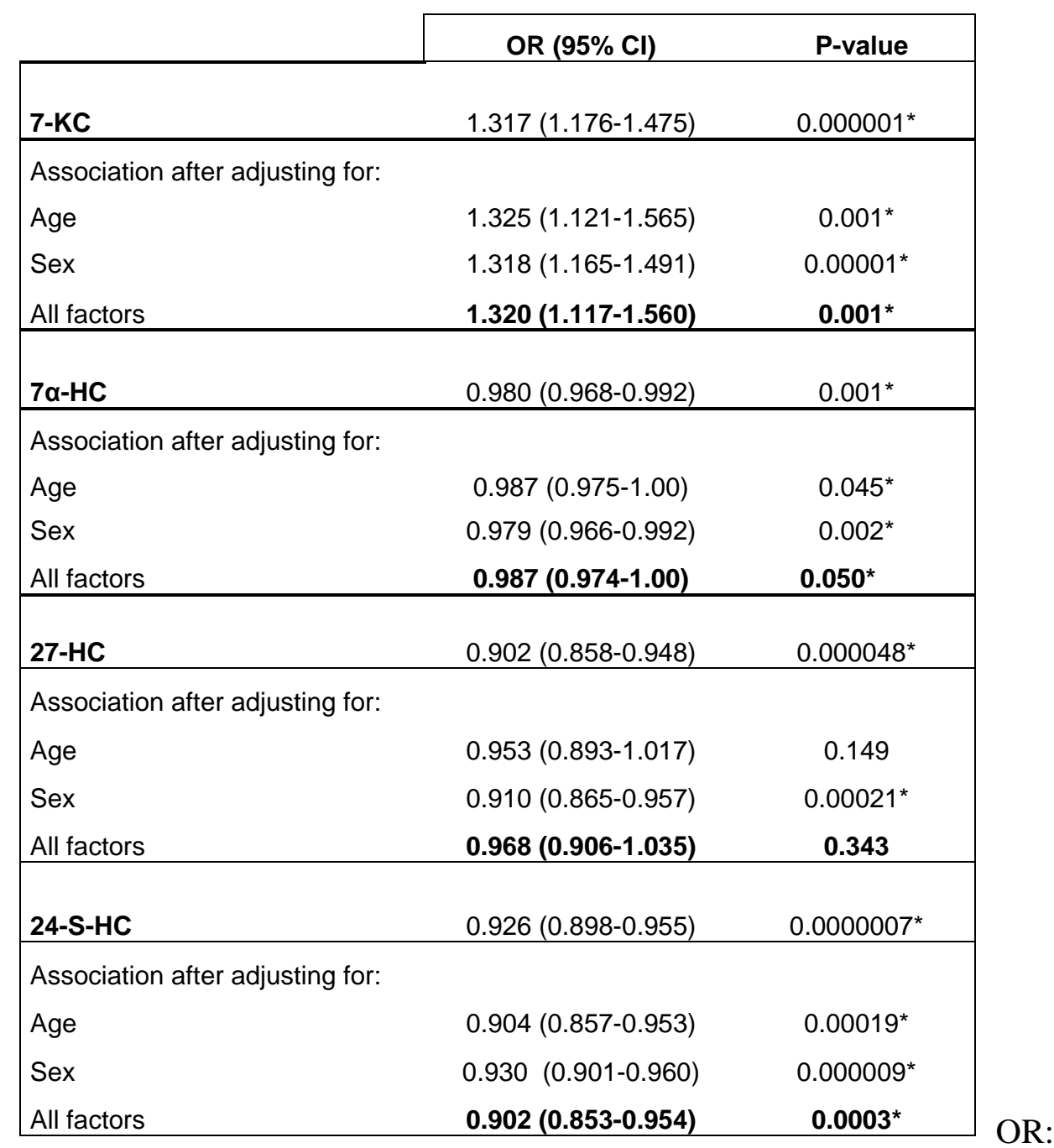

Odd Ratio; $95 \%$ CI: 95 confidence interval. $* \mathrm{P} \leq 0.05$ vs. Donors: statistically significant. 